\title{
Weaker than you might imagine: Determining imageability effects on word recognition
}

\author{
Agata Dymarska ${ }^{1}$, Louise Connell ${ }^{1,2}$, and Briony Banks ${ }^{1}$ \\ ${ }^{1}$ Department of Psychology, Lancaster University \\ ${ }^{2}$ Department of Psychology, Maynooth University
}

\begin{abstract}
Author Note
Agata Dymarska https://orcid.org/0000-0003-0931-3081

Louise Connell https://orcid.org/0000-0002-5291-5267

Briony Banks https://orcid.org/0000-0003-3666-0222
\end{abstract}

This work was supported by the European Research Council (ERC) under the European Union's Horizon 2020 research and innovation programme (grant agreement 682848) to LC.

Correspondence concerning this article should be addressed to Agata Dymarska, Department of Psychology, Fylde College, Lancaster University, Lancaster LA1 4YF, UK. Email:

a.dymarska@lancaster.ac.uk

Open access note: All code and data used in this article are licensed under a Creative Commons Attribution 4.0 International License (CC-BY), which permits use, sharing, adaptation, distribution and reproduction in any medium or format, so long as you give appropriate credit to the original authors and source, provide a link to the Creative Commons license, and indicate if changes were made. To view a copy of the license, visit http://creativecommons.org/licenses/by/4.0/. Data and analysis code can be found at https://osf.io/g3uhp/. 


\begin{abstract}
Imageability - the ease of generating a mental image for a word - has been commonly used as a predictor of word recognition but its effects are highly variable across the literature, raising questions about the robustness and stability of the construct. We compared six existing imageability norms in their ability to predict RT and accuracy in lexical decision and word naming across thousands of words. Results showed that, when lexical and sensorimotor sources of variance were partialled out, imageability predicted little unique variance in word recognition performance and effect sizes varied greatly between norms. Further analysis suggested that such heterogenous effect sizes are likely due to inconsistent strategies in how participants interpret and rate imageability in norming studies despite consistent instruction. Our findings suggest that the ease of generating a mental image for a word does not reliably facilitate word recognition and that imageability ratings should be used with caution in such research.
\end{abstract}

Keywords: Imageability; Sensorimotor information; Word recognition; Situated simulation 


\section{Weaker than you might imagine: imageability effects on word recognition are small and unreliable}

Some words are easier to image perceptually than others. For example, "courthouse" refers to a building or "teacher" refers to a person, both of which can evoke a clear mental image; but "justice" or "knowledge" do not have a direct, tangible referent to image in the same way. This distinction is captured by imageability, which is the ease of generating a mental image associated with a word (e.g., Paivio et al., 1968). Many studies have shown that imageability helps with recognising words (e.g., Balota et al., 2004; de Groot, 1989; Juhasz et al., 2011), alongside the lexical characteristics that facilitate word recognition (high-frequency, early-acquired, and short words are processed quickly and accurately; see Brysbaert et al., 2018 for review). For example, words higher in imageability are recognised faster (than low imageability words) particularly when they are low frequency (Cortese \& Schock, 2013; González-Nosti et al., 2014), acquired later (Cortese \& Khanna, 2007; Cortese \& Schock, 2013; González-Nosti et al., 2014), have atypical spelling (Evans et al., 2012; Woollams, 2015), or small phonological neighbourhood (Westbury \& Moroschan, 2009). The facilitation of word processing by imageability is in line with dual coding theory, where concrete words which can be directly linked with a mental image are recognised and remembered more easily than abstract words that lack such direct connections (Paivio, 1971; 1990; Sadoski \& Paivio, 2004). It is also in line with the theory of semantic richness, where the richer the semantic representation is for a particular word (e.g., greater semantic neighbourhood density, higher number of features, more emotional valence etc.), the easier it is to recognise or remember that word (Buchanan et al., 2001; Pexman et al., 2008; Yap et al., 2012).

Nonetheless, the effect of imageability on word recognition is not as consistent across the literature as one might expect. For example, sometimes imageability explains both speed and accuracy of lexical decision performance (Balota et al., 2004; Yap et al., 2012), but sometimes it cannot explain accuracy (Yap et al., 2015), RT (Yates, 2005), or indeed either measure (Sabsevitz et al., 2005). Sometimes imageability contributes to word naming performance in young adults (Balota et al., 2004), but sometimes it does not (Buchanan et al., 2001). When age of acquisition is controlled, imageability still sometimes exerts an effect on both lexical decision and word naming (Cortese \& 
Schock, 2013), but other times its effect disappears in word naming (Cortese \& Khanna, 2007), lexical decision (Siakaluk et al., 2016), or even in both tasks (Brysbaert et al., 2000). In word naming, Bakhtiar and Weekes (2015) found that the facilitatory effect of early-acquired words was stronger for low-imageability words, while Wilson et al. (2013) found that this effect appeared only with highimageability words. Some of this variability in imageability effects may be due to different choices in experimental designs. For example, relatively small sample sizes, particularly in factorial designs (e.g., 40-60 words per condition: Evans et al., 2012; Yap et al., 2015), may artificially inflate imageability effects if items are sampled from extreme ends on the imageability scale due to the ability of outliers to skew results (e.g., see Ellis \& Monaghan, 2002) or if there is an overrepresentation of items that are more likely to elicit imageability effects (e.g., nouns: see below). Additionally, imageability correlates with a wide number of other lexical and semantic variables, which leads to the possibility of imageability effects being inflated or suppressed according to which variables have been controlled across conditions (in factorial designs) or included as baseline covariates (in regression designs). As a result, the effect that imageability has on word recognition, independent of a wide range of potential confounding variables, across a large sample of thousands of words, remains to be properly quantified.

However, there are patterns of findings across the literature that suggest imageability does not always reflect what it is supposed to (i.e., ease of generating a mental image) and - perhaps as a result - its effects on word recognition are not always consistent. Imageability should reliably measure the ease of generating a mental image for all types of words that can lead to the generation of perceptual imagery. For example, it seems relatively easy to produce a vivid mental image of the verb "to snow" (e.g., a mass of white snowflakes falling endlessly from a dark sky), and just as easy to produce a mental image for the noun "snow" (e.g., drifts of new-fallen snow). Nonetheless, imageability ratings for verbs are lower than for nouns, even in the case of noun-verb homonyms like snow (Bird et al., 2001; Simonsen et al., 2013), and the highest-imageability words in the English language are exclusively nouns (Chiarello et al., 1999). Moreover, Chiarello and colleagues found that, for nouns, the time required to generate an imageability rating decreased as the rating increased (i.e., higher 
imageability $=$ faster rating: $r=-.75$, over $56 \%$ shared variance), which is consistent with the idea that imageability indexes the effort of generating a mental image. For verbs, however, the relationship was much weaker ( $r=-.37$, only $14 \%$ shared variance), suggesting that imageability ratings for verbs are not necessarily indexing imaging effort, or at least not entirely. Imageability ratings also vary for other word types: Clark and Paivio (2004) report a correlation of $r=.67$ with Paivio et al.'s (1968) ratings for adjectives, which reflects only $45 \%$ of shared variance between two sets of ratings derived from identical instructions. Such findings raise the question of validity: do imageability ratings reflect ease of generating a mental image (i.e., its intended construct) only for nouns? And if so, are imageability effects on word recognition an artefact of our experience with noun concepts rather than a semantic effect that applies equally across all parts of speech? If this is the case, then the overall effects of imageability found in the literature might be overestimated if the sample of items contains predominantly nouns.

Although conceptual representations involve perceptual and action experience/information from multiple dimensions (Barsalou et al., 2008; Connell \& Lynott, 2014a; Vigliocco et al., 2009), research on imageability, as well as the lay meaning of imageability, has tended to focus on visual experience and neglect other important sensorimotor information. Imageability norming studies instruct participants to rate how easily a word arouses a mental image ("a mental picture, sound, or other sensory experience": Paivio et al., 1968), and the word "image" or "imagery" itself is highly associated with visual experience (Lynott et al., 2020). Hence, even when an imageability rating study instructs its participants to consider all perceptual information, the ratings tend to be visually biased rather than capturing all perceptual modalities (Connell \& Lynott, 2012a; Speed \& Brysbaert, 2021). For example, Connell and Lynott (2012a) found that imageability ratings are negatively correlated with the strength of auditory and gustatory experience of a concept (i.e., more strongly auditory or gustatory = lower imageability), indicating that sound and taste are not well captured by imageability, even though people are able to generate imagery in any modality when asked to do so (e.g., Palmiero et al., 2009). More specifically, the consciously-available perceptual information that informs a single imageability rating may not correspond to the sum of multiple ratings of individual modalities, as 
people tend to distort or neglect all but the dominant modality when attempting to evaluate sensory experience as a whole (Connell \& Lynott, 2016a). While most words in English are indeed dominated by visual experience (Lynott et al., 2020), other sensorimotor dimensions are also critical, particularly for abstract concepts where modalities like hearing (Connell \& Lynott, 2012a) and interoception (i.e., sensations inside the body: Connell et al., 2018) are more important than for concrete concepts. It is therefore unlikely that imageability is accurately capturing useful sensorimotor information about words whose referent concepts are dominated by dimensions other than vision, such as toothache, murmur, doubt, or bland. This brings back the question of construct validity: are imageability effects on word recognition limited to visual images rather than applying generally to all forms of sensorimotor experience? If this is the case, then imageability effects in the literature may depend on the sample of items containing a clear visual vs. non-visual distinction rather than an overall sensorimotor vs. non-sensorimotor distinction.

Such reliance on vision also represents a problem for the interpretation of imageability effects because - separate to the notion of imageability - words are processed faster when their referents can be visually perceived (Connell \& Lynott, 2014b; Chedid et al., 2019; Estes et al., 2015). One reason for this facilitation is that directing one's attention to a particular sensory modality pre-activates the relevant sensory cortex (Foxe et al., 2005; Langner et al., 2011) which facilitates processing semantic information in that modality (Connell \& Lynott, 2012b; Connell et al., 2012; see also Spence et al., 2001). Hence, the visual attention required by a visual word recognition task facilitates representing visual information in the referent concept, which in turn contributes to the processes that drive word recognition so that strongly-visual words are recognised more quickly and easily (Connell \& Lynott, 2014b, 2016b). If imageability ratings are mainly driven by visual information, then how should we interpret their effects on word recognition: are they at least in part attributed to facilitation by attentional modulation in a visual task? It is important to determine the extent to which imageability itself - that is, a construct that measures the ease of generating conscious imagery of a word facilitates word recognition independently of visual attentional modulation. 
More broadly, sensorimotor grounding (i.e., the perceptual and action experience underlying a referent concept) and imagery are different theoretical constructs, the former concerned with unconscious, automatic activation of sensorimotor systems and the latter concerned with conscious evaluation or manipulation of sensorimotor information, and it is important to distinguish them. However, it is possible that imageability ratings might simply measure some sensorimotor grounding rather than imagery per se (i.e., the ease of consciously generating imagery for a word). Studies showing that sensorimotor strength (Connell \& Lynott, 2012a; see also Speed \& Brysbaert, 2021) or relative embodiment (degree to which the meaning of a verb involves a human body; Sidhu et al., 2014) outperforms imageability in predicting word recognition suggest that it is the sensorimotor grounding of a word that facilitates its recognition rather than the ease of consciously generating imagery for the word. Moreover, findings that perceptual facilitation effects on word recognition are modality-specific and vary by task (i.e., depending on how perceptual attention is implicitly directed by lexical decision task vs. word naming: Connell \& Lynott, 2014b) further support the idea that such facilitation in word recognition arises from grounding word meaning in perceptual and action systems rather than from conscious imagery processes. Nonetheless, other work suggests that the ability of perceptual strength to outperform imageability in predicting word recognition performance may depend on the language examined (Vergallito et al., 2020), the baseline lexical model (Khanna \& Cortese, 2021), or whether perceptual strength ratings in multiple modalities are aggregated in a single composite variable (Speed \& Brysbaert, 2021). It is therefore important to determine the extent to which imageability itself - that is, a construct that measures the ease of generating conscious imagery of a word - facilitates word recognition independently of sensorimotor grounding.

A final possible reason for variable imageability effects across the literature is that imageability ratings themselves can vary enormously across different sets of norms, therefore imageability itself (i.e., the ease of consciously generating imagery for a word) may not always be a reliable and stable construct, despite using the same method and instructions from Paivio et al. 
$(1968)^{1}$. For example, on the 1-7 imageability scale, the following words easily evoke mental images according to one set of norms but not another: "norm" (2.5 in Cortese \& Fugett, 2004; 6.0 in Toglia \& Battig, 1978), "head" (2.4 in Bird et al., 2001; 6.8 in Cortese \& Fugett, 2004) and "spire" (2.7 in Cortese \& Fugett, 2004; 5.5 in Scott et al., 2018). Indeed, previous work that has systematically examined the stability of imageability ratings has sometimes found major discrepancies between participant samples (e.g., Campos \& Sueiro, 1991; Grandy et al., 2020). While newer imageability norms report consistency with existing ones in method and ratings (e.g., Bird et al. 2001; Cortese \& Schock, 2013; Scott et al., 2018), they nonetheless vary in terms of participants' age and country of testing, word samples and rating distributions, as well as testing methods (see Table 1 for an overview of the norms used in the current study). For instance, Campos \& Sueiro (1991) correlated imageability ratings across age and sex, and found that while some subgroups rated imageability consistently (e.g., $r=.94$ for men vs. women aged 21-40), others did not (e.g., $r=.14$ for men vs. women aged 41-60; $r$ $=.33$ for adults age $21-40$ vs. 41-60). Clearly, whatever the reason for such discrepancies (e.g., age, sex, cultural change over time, geographic location), it is clear that different samples of participants sometimes base their imageability ratings on qualitatively different information, which in turn may influence the efficacy of those ratings in predicting word recognition performance. It is therefore important to examine a range of sources of imageability ratings when attempting to establish the effect that the ease of generating mental imagery has on word recognition performance, and hence whether imageability effects can be considered reliable.

\section{Current Study}

In the present paper, our goal was to examine the construct of imageability, what it measures, and the extent to which it reliably contributes to word recognition performance. To this end, we compared a number of different sets of imageability norms that spanned a number of different word classes in a series of exploratory studies across several different megastudy datasets of lexical decision and word naming. As

\footnotetext{
${ }^{1}$ Any word which, in your estimation, arouses a mental image (i.e., a mental picture, or sound, or other sensory experience) very quickly and easily should be given a high imagery rating; any word that arouses a mental image with difficulty or not at all should be given a low imagery rating (Paivio et al., 1968, p. 4).
} 
outlined above, one possible reason for the variability in published imageability results is low consistency in the baseline lexical variables examined or controlled alongside imageability. In Study 1, we therefore examined whether different sources of imageability norms produce consistent effects in word processing when the baseline (lexical) model was held constant. Results remained highly variable, with imageability ratings ranging from non-existent to large, depending on the source of the imageability norms used.

Hence, in Study 2, we explored what possible aspects of the imageability norms might explain their variability. From a large set of candidate variables, we obtained 6 lexical and sensorimotor components through principal components analysis (PCA) and used them to predict imageability ratings from different norms. We found that variance in imageability ratings was primarily explained by a sensorimotor component that reflected object manipulability (e.g., incorporating visual and haptic strength, hand/arm action, and word class as a noun), while other effects varied somewhat between different sets of imageability norms. These results suggest that much of the variance in imageability ratings is attributable to sensorimotor grounding of the referent concept rather than ease of consciously generating a mental image per se. Nonetheless, the variability between different norms remained.

In Study 3, we therefore used the lexical-sensorimotor PCA components as predictors of lexical decision and naming performance for the subsets of words covered by each set of imageability norms. All components predicted performance, but variably for different subsets of words. Next, Study 4 examined the extent to which "pure" imageability (i.e., the ease of generating a mental image, distinct from lexical variables and sensorimotor grounding of the referent concept) affected word recognition performance over and above the lexical variables and the sensorimotor components of Study 3. We found that the results were still quite inconsistent, with some imageability norms producing large effects, yet others not eliciting any effects on word recognition at all. Finally, in Study 5, we conducted an internal meta-analysis to determine the overall effect size of imageability on word recognition, which showed high heterogeneity between different sets of imageability norms that could be only partially explained by a moderating effect of dependent measures. The meta effect size of imageability on word recognition was very small, explaining only $0.30 \%$ of the variance in performance. These results suggest that imageability is not, in fact, a stable construct that reliably predicts of semantic effects in word processing. 


\section{Data Availability Statement}

All data and analysis code for the studies can be found at: https://osf.io/g3uhp/

\section{Study 1 - Does imageability consistently contribute to word recognition?}

In this study, we analysed 6 different sources of imageability norms in their ability to predict word recognition. Using lexical decision and word naming performance from the English Lexicon Project (Balota et al., 2007) and lexical decision data from the British Lexical Project (Keuleers et al., 2012), we ran hierarchical regression analyses with a consistent, data-driven lexical baseline model, to compare how well different sources of imageability predicted word recognition performance. Based on previous literature (e.g., Cortese \& Schock, 2013), we expected imageability to facilitate accuracy and RT of word recognition. Additionally, we expected the effects to be stronger for low frequency words (i.e., imageability*frequency interaction) because active semantic content facilitates the processing of orthographic and/or phonological representations during visual word recognition (e.g., Coltheart et al., 2001; Harm \& Seidenberg, 2004). When a written word is presented, its orthographic representation spreads activation to semantic content, which in turn feeds activation back to the orthographic and/or phonological representation and facilitates a relatively rapid task response (i.e., "yes" in lexical decision; correct pronunciation in word naming). However, such semantic facilitation will not occur if the orthographic or phonological representations independently reach a sufficiently high level of activation to enable a task response, which most often happens for common wordforms. As a result, low-frequency words like "cad" benefit more from semantic facilitation than do highfrequency words like "cat” (e.g., Connell \& Lynott, 2016; de Groot, 1989; James, 1975). If imageability facilitates word processing, and does so more strongly for low-frequency words, then we would expect a pattern of negative part correlations for imageability and positive part correlations for its interaction with frequency in the RT analysis, and the reverse in the accuracy analysis. 


\section{Method}

\section{Materials}

Data selection. We used imageability ratings from 6 sources, which we term the Bird norms (Bird et al., 2001); Bristol norms (Stadthagen-Gonzalez \& Davis, 2006); Chiarello norms (Chiarello et al., 1999); Cortese norms (Cortese \& Fugett, 2004; Schock et al., 2012); Glasgow norms (Scott et al., 2018); MRC norms (Coltheart, 1981, combining data from multiple imageability datasets including Gilhooly \& Logie, 1980; Paivio et al., 1968; Toglia \& Battig, 1978). We combined Cortese and Fugett (2004) and Schock et al. (2012) into one larger set of norms, because they were obtained by the same research group with very similar methods and populations, covering monosyllabic and disyllabic words, respectively. Critically, all imageability norming studies used the same instructions to describe imageability to participants and the same 7-point rating scale (originating with Paivio et al., 1968).

Table 1 shows an overview of the norming studies.

Table 1: Overview of the imageability norms used in the current studies

\begin{tabular}{llllll}
\hline $\begin{array}{l}\text { Imageability } \\
\text { norms }\end{array}$ & $\begin{array}{l}\text { Publication } \\
\text { year }\end{array}$ & Country of origin & $\begin{array}{l}\text { Mean participant } \\
\text { age (years) }\end{array}$ & $\begin{array}{l}\text { Number of } \\
\text { words }\end{array}$ & Types of word \\
\hline Bird & 2001 & UK & 65.0 & 2645 & All Types \\
Bristol & 2006 & UK & 19.7 & 1526 & Nouns \& Verbs \\
Chiarello & 1999 & USA & 20.7 & 1197 & Nouns \& Verbs \\
Cortese & $2004 \& 2012$ & USA & 23.8 & 6000 & All Types \\
Glasgow & 2018 & UK & 21.7 & 5500 & Nouns, Verbs, \\
MRC & 1960s-1980s & UK+USA+Canada & Unknown & 9240 & $\begin{array}{l}\text { Adjectives, \& Adverbs } \\
\end{array}$ \\
\hline
\end{tabular}

We collated lexical decision accuracy and response time (RT) data from lexical decision tasks (LDT) in the British Lexicon Project (BLP, Keuleers et al., 2012) and the English Lexicon Project (ELP, Balota et al., 2007), as well as accuracy and RT from a word naming task in the ELP, for use as dependent variables (DVs). Our dataset therefore comprised the combination of imageability ratings (from one or more sets of norms) and word recognition DVs, which resulted in a sample of 9789 
words: 9554 words had Lexical Decision and Word Naming Accuracy and RT data from the ELP, and 7550 words had Lexical Decision Accuracy and RT data from the BLP. Note that the different sets of imageability norms covered quite different samples of words, with only 19 words present across all norms, so we analysed them separately; a full list of word samples for each set of imageability norms is available in supplemental materials. R tidyverse package (Wickham et al., 2019) was used to combine datasets.

Null lexical model selection. While variables like word frequency, length or phonological neighbourhood are often included in null (i.e., baseline) lexical decision models (Connell \& Lynott, 2012a, Cortese et al., 2010, 2015; Yap et al., 2012), we wanted to select a lexical model that provided optimal fit to the current data. We did not include age of acquisition, as there is some disagreement as to whether it is a lexical or a lexico-semantic variable (e.g., Cortese \& Khanna, 2007; Zevin \& Seidenberg, 2002). Many of our candidate lexical predictors were obtained from the ELP: word length in letters, $\log$ subtitle frequency (LgSUBTLWF), log subtitle contextual diversity (LgSUBTLCD), Orthographic Levenshtein Distance (OLD), Phonological Levenshtein Distance (PLD), and number of syllables. We also included prevalence, which is another way of estimating frequency by measuring how many participants know a particular word (Brysbaert et al., 2018), and Zipf frequency (van Heuven et al., 2014), which is a standardised measure of word frequency that can outperform simple $\log$ frequency. From this set of lexical variables, we objectively selected a baseline lexical model which provided the best fit to the word recognition data.

We ran a Bayesian regression model in JASP (0.14.1: JASP Team, 2020), with 8 possible candidate variables (word length, number of syllables, OLD, PLD, LgSUBTLWF, LgSUBTLCD, Zipf frequency, prevalence) as predictor variables, and accuracy and RT from each BLP and ELP dataset as dependent variables. For each dependent variable, we selected those predictors where evidence for their inclusion was $\mathrm{BF}_{10}>3$ (detailed analysis is available in supplemental materials); that is, the model explained more variance in word recognition performance when the variable was included, than when it was not. After selecting the best candidate model for each analysis, we combined the best predictors from RT and accuracy per task, and made sure that the model with all 
selected predictors offered the strongest evidence (i.e., the highest $R^{2}$ and $\mathrm{BF}_{10}$ ) over the null in predicting accuracy and RT performance. We then combined the predictors from all three tasks (BLP lexical decision, as well as ELP lexical decision and word naming), and again made sure that for each $\mathrm{DV}$, the inclusion of the selected predictors offered the highest improvement over the null. The final null model consisted of: word length, number of syllables, LgSUBTLCD, OLD, and prevalence.

Table 2 shows the characteristics of each predictor across the samples of words featured in each set of imageability norms, which were mostly consistent across norms except for the number of words per sample. However, there were some differences between tasks. First, there were slightly more words in the ELP dataset than in the BLP dataset, especially for the Bird, Glasgow and MRC norms. For these norms, the ELP words were on average 1 letter and 0.5 syllables longer than the BLP words. Participants also responded to the words from the ELP around 100ms slower than to the words from the BLP. 
Table 2: Mean values (with standard deviations in parentheses) of lexical characteristics and word recognition measures from the British Lexicon Project (BLP) and English Lexicon Project (ELP) for the words covered by each set of imageability norms in Study 1.

\begin{tabular}{|c|c|c|c|c|c|c|c|}
\hline Dataset & Variable & Bird & Bristol & Chiarello & Cortese & Glasgow & MRC \\
\hline BLP (RT \& & $\mathrm{N}$ & 1081 & 1496 & 1129 & 5720 & 3347 & 3346 \\
\hline \multirow[t]{8}{*}{ Accuracy) } & Length & $5.51(1.48)$ & $5.39(1.06)$ & $5.00(0.95)$ & $5.27(1.34)$ & $5.54(1.38)$ & $5.30(1.47)$ \\
\hline & Syllables & $1.55(0.51)$ & $1.53(0.50)$ & $1.47(0.50)$ & $1.49(0.50)$ & $1.57(0.50)$ & $1.49(0.51)$ \\
\hline & OLD & $1.94(0.57)$ & $1.90(0.46)$ & $1.78(0.37)$ & $1.89(0.52)$ & $1.96(0.57)$ & $1.88(0.57)$ \\
\hline & LgSUBTLCD & $2.53(0.84)$ & $2.36(0.63)$ & $2.27(0.70)$ & $2.30(0.75)$ & $2.46(0.69)$ & $2.48(0.82)$ \\
\hline & Prevalence & $2.23(0.35)$ & $2.26(0.28)$ & $2.21(0.32)$ & $2.16(0.37)$ & $2.29(0.25)$ & $2.22(0.37)$ \\
\hline & Imageability & $4.29(1.08)$ & $3.94(1.37)$ & $4.80(1.36)$ & $4.38(1.44)$ & $4.84(1.35)$ & $4.70(1.02)$ \\
\hline & LD RT & $578(63)$ & $582(53)$ & $589(57)$ & $597(65)$ & $575(52)$ & $581(62)$ \\
\hline & LD Accuracy & $0.94(0.130)$ & $0.95(0.08)$ & $0.93(0.12)$ & $0.91(0.15)$ & $0.96(0.07)$ & $0.93(0.14)$ \\
\hline ELP (RT \& & $\mathrm{N}$ & $1434 / 1435$ & 1502 & 1186 & 5820 & 4448 & 4572 \\
\hline \multirow[t]{10}{*}{ Accuracy) } & Length & $6.41(2.21)$ & $5.39(1.06)$ & $5.03(0.95)$ & $5.28(1.34)$ & $6.34(2.01)$ & $6.27(2.25)$ \\
\hline & Syllables & $1.99(0.96)$ & $1.53(0.50)$ & $1.53(0.56)$ & $1.49(0.50)$ & $1.98(0.90)$ & $1.98(0.99)$ \\
\hline & OLD & $2.29(0.88)$ & $1.90(0.46)$ & $1.80(0.38)$ & $1.90(0.52)$ & $2.25(0.79)$ & $2.22(0.83)$ \\
\hline & LgSUBTLCD & $2.38(0.88)$ & $2.36(0.63)$ & $2.27(0.70)$ & $2.29(0.75)$ & $2.37(0.69)$ & $2.34(0.83)$ \\
\hline & Prevalence & $2.21(0.36)$ & $2.26(0.27)$ & $2.20(0.32)$ & $2.15(0.38)$ & $2.28(0.25)$ & $2.20(0.38)$ \\
\hline & Imageability & $4.20(1.11)$ & $3.94(1.37)$ & $4.80(1.36)$ & $4.38(1.44)$ & $4.70(1.36)$ & $4.55(1.04)$ \\
\hline & LD RT & $681(98)$ & $660(72)$ & $665(72)$ & $678(84)$ & $674(85)$ & $682(96)$ \\
\hline & LD Accuracy & $0.93(0.12)$ & $0.938(0.096)$ & $0.93(0.10)$ & $0.90(0.14)$ & $0.95(0.09)$ & $0.93(0.13)$ \\
\hline & WN RT & $652(76)$ & $637(56)$ & $632(50)$ & $642(61)$ & $648(66)$ & $652(74)$ \\
\hline & WN Accuracy & $0.97(0.06)$ & $0.98(0.04)$ & $0.98(0.05)$ & $0.97(0.06)$ & $0.98(0.04)$ & $0.97(0.07)$ \\
\hline
\end{tabular}

Note: In the Bird norms, one word was missing an RT value for ELP lexical decision. This did not affect descriptive statistics, so all word statistics for ELP are reported together.

\section{Design and analysis}

For each set of imageability rating norms, we conducted a hierarchical regression model to see how well the imageability norms predicted accuracy and zRT for lexical decision and word naming tasks from BLP and ELP. All interacting variables were centred. In Step 1 we entered all the lexical predictors selected above, in Step 2 we added imageability (each set of norms was analysed separately), and in Step 3 we added the imageability*frequency interaction using prevalence as a proxy for frequency (as it was the best-performing frequency measure in the null model selection), which allowed us to ascertain whether the contribution of imageability varied with word frequency. There were 36 regression models in total, representing six different imageability norms (Bird, Bristol, 
Chiarello, Cortese, Glasgow, MRC) by six different dependent variables (BLP LDT RT, BLP LDT accuracy, ELP LDT RT, ELP LDT accuracy, ELP Naming RT, ELP Naming accuracy).

We ran Bayesian linear regressions in JASP (JASP Team, 2020) with default JZS priors $(r=$ .354) and a Bernoulli distribution $(p=0.5)$, from which we report Bayes Factors for model comparisons between hierarchical steps and posterior inclusion Bayes Factors of imageability coefficients (i.e., indicating the relative likelihood of models including a particular predictor compared to models excluding it). In addition, to calculate the unique contribution each predictor makes to the dependent measure in question, we ran NHST linear regression analyses using the same structure as the Bayesian linear regression and took part correlation coefficients for each predictor from the Step 3 model.

\section{Results \& Discussion}

Zero-order correlations between predictors and DVs are available in supplemental materials, along with full details of the regression models.

\section{Lexical (null) model}

As shown in Table $2, R^{2}$ for lexical decision accuracy varied across different sets of imageability norms, and even more so between different word recognition tasks. Depending on the task and imageability dataset, lexical models explained between $21.6 \%$ to $72.3 \%$ variability in word processing. The lexical model's contribution was lowest for word naming, as typically occurs in analysis of visual word recognition (e.g., Balota et al., 2004): 21.6\% to 41.6\% variance for accuracy, and between $23.7 \%$ and $53.4 \%$ for RT. For lexical decision, the model performed similarly for BLP and ELP data, with accuracy having a wider range (43.2\% - 72.3\%) than RT (55.4\% - 70.3\%).

\section{Imageability}

Change in $\mathrm{R}^{2}$ for the Step 2 model with imageability (Tables 3 and 4) shows that imageability contributed up to $1.8 \%$ of additional variance over and above the lexical variables included in the null model, depending on the task and dataset. 
However, the imageability effect size was highly variable across different sets of norms for both accuracy and RT. The Cortese and MRC imageability norms were the most reliable in terms of eliciting facilitation effects in both lexical decision and word naming DVs, followed by the Bird norms, although the effects were at times very small (e.g., less than $1 \%$ of variance). Other norms performed inconsistently, and produced effects in only three of the six DVs (i.e., not in word naming or in one accuracy variable). Indeed, imageability had no effect in approximately one third of analyses (11 out of 36 models).

There were also systematic differences between tasks and between dataset sources. The effects were larger for accuracy than RT in word naming, but smaller for accuracy than RT in lexical decision (see standardised regression coefficients and part correlations in supplementals). Overall, imageability effect sizes were smaller for word naming than for lexical decision, consistent with previous findings in the literature (e.g., Ferrand et al. 2011); indeed, half of the norms (Bristol, Chiarello and Glasgow norms) failed to produce any effects on naming at all. Within lexical decision, imageability effects were generally larger for data from the BLP than the ELP, in particular for the norms which originated from UK participants (Bird, Bristol and Glasgow norms).

\section{Imageability*frequency}

The Step 3 model revealed that an imageability*frequency interaction appeared in the majority of analyses (24 out of 36 models) and explained up to $4.9 \%$ of variance on top of imageability alone, but the size and indeed direction of the interaction effect varied enormously between norms and datasets (see Tables 3 and 4). Indeed, only half of all analyses (18 out of 36 models) elicited interaction effects in the expected direction.

While we expected imageability to produce stronger facilitation effects for low-frequency words, Figure 1 shows that no set of norms did so consistently for both accuracy (i.e., negative part correlation for interaction term) and RT (i.e., positive part correlation for interaction term). Although the Cortese norms produced an interaction effect on all DVs, its effects on RT were unexpectedly negative; that is, imageability elicited a larger facilitatory effect on high frequency words in all RT 
datasets, contrary to predictions. Other imageability norms also showed inconsistent interactions, typically boosting facilitation of low-frequency words at least sometimes for accuracy but with nonexistent or reverse direction (i.e., stronger facilitation for high-frequency words) effects on RT. The Bristol norms performed the worst overall, producing an interaction effect in the expected direction in only 2 of the 6 DVs: BLP lexical decision accuracy and RT. The other norms elicited an interaction effect in the expected direction in 3 or 4 out of the 6 DVs.

There were some systematic patterns in the imageability*frequency interaction effects across different word recognition tasks, but they were overall weaker and more inconsistent than for initial imageability effects in Step 2. The interaction effects on accuracy were strongest for the BLP lexical decision dataset, where all 6 imageability norms produced facilitation for low-frequency words, as expected. However, in the ELP lexical decision dataset, 4 sets of norms had the expected interaction effect on accuracy, which was fewer than in word naming, where 5 out of 6 sets of norms elicited the expected effect on accuracy. Regardless of task, few of the norms produced the expected interaction for RT, although several produced interaction effects in the opposite direction (i.e., imageability had stronger facilitation for high-frequency words than low-frequency words). This pattern of effects did not support previous findings that imageability has a reliably stronger facilitatory effect on the latency of recognising low frequency words (Cortese \& Schock, 2013; González-Nosti et al., 2014), although it does for accuracy of recognition. 
Table 3: Variance in word recognition accuracy explained by each step of the Study 1 regression model (change in $R^{2}$, with Bayes Factors for each step compared to the previous) and uniquely explained by imageability and its frequency interaction in the Step 3 model (squared part correlations), for each set of imageability norms.

\begin{tabular}{llllllll}
\hline Step & Parameter(s) & Bird & Bristol & Chiarello & Cortese & Glasgow & MRC \\
\hline BLP Lexical Decision & & & & & & \\
1 & Null model $R^{2}$ & $.671^{* * *}$ & $.490^{* * *}$ & $.619^{* * *}$ & $.684^{* * *}$ & $.432^{* * *}$ & $.723^{* * * *}$ \\
2 & Imageability $\Delta R^{2}$ & $.012^{* * *}$ & $.011^{* * *}$ & .001 & $.002^{* * *}$ & $.006^{* * * *}$ & $.005^{* * * *}$ \\
3 & Imageability*frequency $\Delta R^{2}$ & $.049^{* * *}$ & $.038^{* * *}$ & $.011^{* * *}$ & $.012^{* * *}$ & $.032^{* * *}$ & $.019^{* * *}$ \\
& Imageability $s r^{2}$ & .025 & .034 & .005 & .005 & .030 & .011 \\
& Imageability*frequency $s r^{2}$ & .049 & .010 & .001 & .012 & .032 & .019 \\
\hline ELP Lexical Decision & & & & & & \\
1 & Null model $R^{2}$ & $.652^{* * *}$ & $.571^{* * *}$ & $.583^{* * *}$ & $.670^{* * *}$ & $.515^{* * * *}$ & $.683^{* * * *}$ \\
2 & Imageability $\Delta R^{2}$ & .002 & .000 & $.007^{* * *}$ & $.010^{* * *}$ & .000 & $.001^{* * *}$ \\
3 & Imageability*frequency $\Delta R^{2}$ & $.004^{* *}$ & .000 & $.028^{* * *}$ & $.025^{* * *}$ & .000 & $.008^{* * *}$ \\
& Imageability $s r^{2}$ & .002 & .000 & .015 & .015 & .000 & .007 \\
& Imageability*frequency $s r^{2}$ & .004 & .000 & .028 & .026 & .000 & .008 \\
\hline ELP & Word Naming & & & & & & \\
1 & Null model $R^{2}$ & $.416^{* * *}$ & $.216^{* * *}$ & $.217^{* * *}$ & $.282^{* * *}$ & $.236^{* * *}$ & $.407^{* * * *}$ \\
2 & Imageability $\Delta R^{2}$ & .001 & .000 & .001 & $.003^{* * *}$ & .001 & $.003^{* * * *}$ \\
3 & Imageability*frequency $\Delta R^{2}$ & $.020^{* * *}$ & .000 & $.013^{* * * *}$ & $.012^{* * *}$ & $.011^{* * *}$ & $.036^{* * *}$ \\
& Imageability $s r^{2}$ & .002 & .000 & .004 & 0.004 & .006 & .007 \\
& Imageability*frequency $s r^{2}$ & .020 & .000 & .013 & 0.012 & .011 & .036 \\
\hline
\end{tabular}

Note. The summed $\mathrm{s} r^{2}$ for imageability and imageability*frequency in the final model can be greater than the corresponding $\Delta R^{2}$ due to mutual suppression of parameters.

$* \mathrm{BF}_{10} \geq 3$, positive evidence; $* * \mathrm{BF}_{10} \geq 20$, strong evidence; *** $\mathrm{BF}_{10} \geq 150$, very strong evidence 
Table 4: Variance in word recognition zRT explained by each step of the Study 1 regression model (change in $R^{2}$, with Bayes Factors for each step compared to the previous) and uniquely explained by imageability and its frequency interaction in the Step 3 model (squared part correlations), for each set of imageability norms.

\begin{tabular}{llllllll}
\hline Step & Parameter(s) & Bird & Bristol & Chiarello & Cortese & Glasgow & MRC \\
\hline BLP lexical Decision & & & & & & \\
1 & Null model $R^{2}$ & $.680^{* * *}$ & $.554^{* * *}$ & $.640^{* * *}$ & $.651^{* * *}$ & $.566^{* * *}$ & $.658^{* * *}$ \\
2 & Imageability $\Delta R^{2}$ & $.017^{* * *}$ & $.018^{* * *}$ & $.008^{* * *}$ & $.008^{* * *}$ & $.014^{* * *}$ & $.010^{* * *}$ \\
3 & Imageability*frequency $\Delta R^{2}$ & .001 & $.007^{* * *}$ & .001 & $.001^{* * *}$ & $.003^{* * *}$ & .000 \\
& Imageability $s r^{2}$ & .018 & .025 & .005 & .008 & .016 & .008 \\
& Imageability*frequency $s r^{2}$ & .002 & .007 & .002 & .000 & .003 & .000 \\
\hline ELP Lexical Decision & & & & & & \\
1 & Null model $R^{2}$ & $.701^{* * *}$ & $.595^{* * *}$ & $.609^{* * *}$ & $.640^{* * *}$ & $.655^{* * *}$ & $.703^{* * * *}$ \\
2 & Imageability $\Delta R^{2}$ & $.007^{* * *}$ & $.005^{* *}$ & $.010^{* * *}$ & $.012^{* * *}$ & $.002^{* * *}$ & $.005^{* * * *}$ \\
3 & Imageability*frequency $\Delta R^{2}$ & $.003^{* *}$ & .001 & .000 & $.002^{* * *}$ & $.001^{*}$ & $.001 * *$ \\
& Imageability $s r^{2}$ & .007 & .002 & .010 & .010 & .001 & .004 \\
& Imageability*frequency $s r^{2}$ & .003 & .001 & .000 & .002 & .001 & .001 \\
\hline ELP Word Naming & & & & & & \\
1 & Null model $R^{2}$ & $.532^{* * *}$ & $.296^{* * *}$ & $.237^{* * *}$ & $.377^{* * *}$ & $.456^{* * *}$ & $.534^{* * *}$ \\
2 & Imageability $\Delta R^{2}$ & $.005^{* *}$ & .000 & .000 & $.002^{* * *}$ & .001 & $.002^{* * * *}$ \\
3 & Imageability*frequency $\Delta R^{2}$ & .000 & .000 & .000 & $.003^{* * *}$ & .000 & $.002^{* * *}$ \\
& Imageability $s r^{2}$ & .004 & .000 & .000 & .001 & .001 & .003 \\
& Imageability*frequency $s r^{2}$ & .001 & .000 & .000 & .003 & .000 & .001 \\
\hline
\end{tabular}

Note. The summed $\mathrm{s} r^{2}$ for imageability and imageability*frequency in the final model can be greater than the corresponding $\Delta R^{2}$ due to mutual suppression of parameters.

$* \mathrm{BF}_{10} \geq 3$, positive evidence; $* * \mathrm{BF}_{10} \geq 20$, strong evidence; *** $\mathrm{BF}_{10} \geq 150$, very strong evidence 

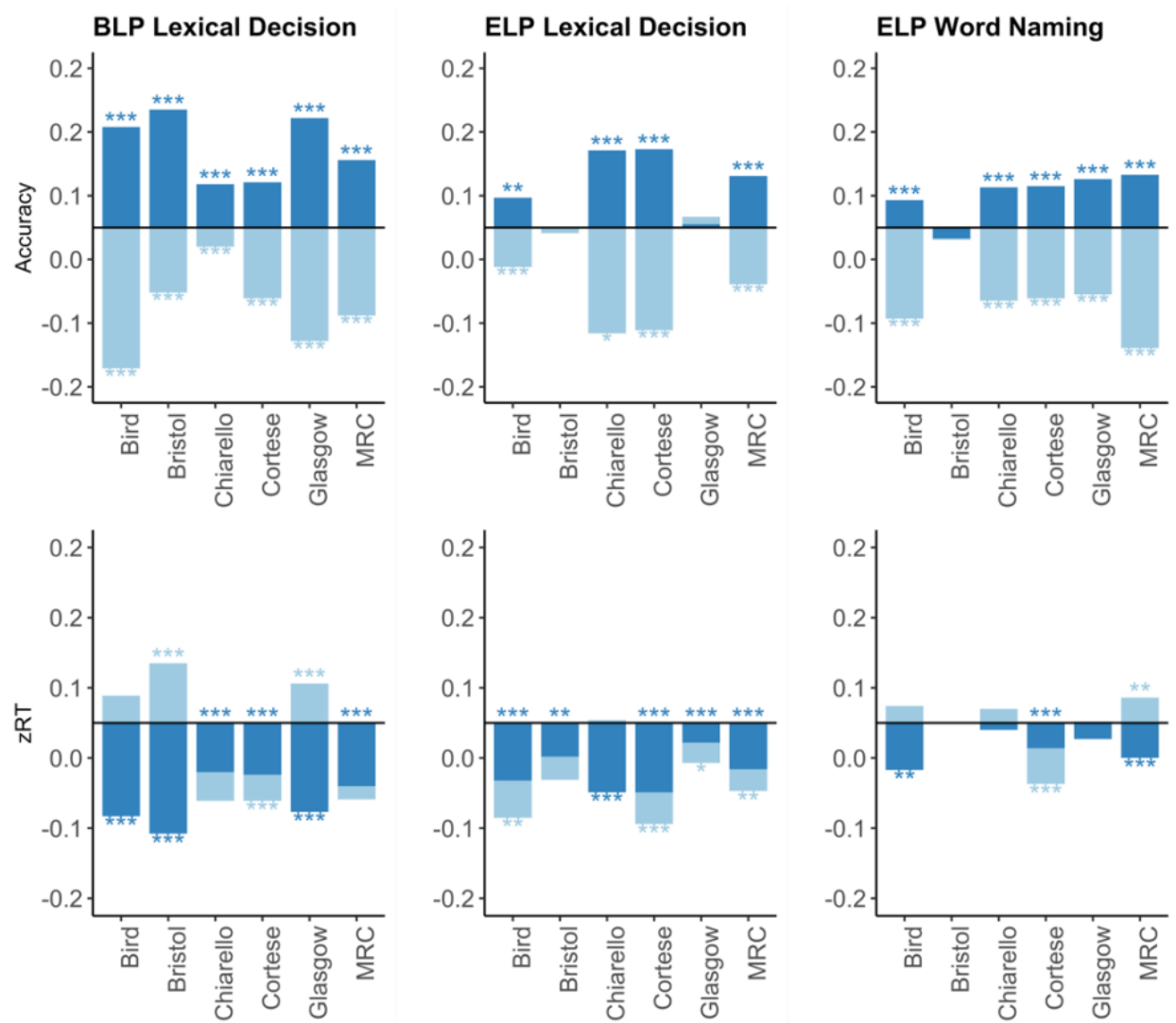

Imageability ${ }^{\star}$ Prevalence

Imageability

Figure 1: Part correlations in the Step 3 (final) regression model of Study 1. Stacked bars represent the combined effect of imageability (darker shade) and the imageability*frequency interaction (lighter shade). Asterisks refer to the Bayes Factor (BF inclusion from Step 3) of the relevant parameter: * $\mathrm{BF}_{\text {inclusion }} \geq 3$, positive evidence; $* * \mathrm{BF}_{\text {inclusion }} \geq 20$, strong evidence; $* * * \mathrm{BF}_{\text {inclusion }} \geq 150$, very strong evidence.

\section{Conclusion}

We found that imageability did not consistently predict word recognition performance, even when the same lexical baseline model was used in the analysis. There were large differences in effect sizes depending on the source of imageability norms used, though where effects did emerge, they were in the expected facilitatory direction. Moreover, imageability did not consistently interact with frequency (represented as word prevalence), again varying with the norms used, and where the interaction emerged there was no consistency as to whether imageability effects were stronger for low-frequency or high-frequency words. This discrepant pattern of results could be due to a number of reasons. Each dataset used different samples of words which could differ in some characteristics that impact on their susceptibility to imageability effects (although Table 2 shows that the norms were 
consistent across many lexical variables). Alternatively, some participants might be more accurate at judging the ease of generating a mental image, and these differences could affect how successful their imageability ratings are at predicting word recognition. Finally, it is also possible that ease of generating mental imagery - that is, the construct imageability is meant to reflect - is not a reliable predictor of word recognition, and any effects found in the literature so far should not be attributed to ease of generating a mental image of a word. Instead, imageability ratings might capture partial aspects of the sensorimotor information that constitutes word meaning, which facilitates word recognition according to the attentional demands of the word recognition task (Connell \& Lynott, 2014b, 2016b). Particularly, since imageability is primarily associated with vision (Connell \& Lynott, 2012a), it cannot explain full sensorimotor processing (i.e., relating to multiple sensory modalities and actions) which may lead to variation of its effects depending on the type of words that are analysed and their sensorimotor content. We address these possibilities in the next study.

\section{Study 2 - Which aspects of imageability norms might explain their variability?}

Study 1 showed that imageability ratings can explain some differences in word recognition performance, but there is large variability in the results depending on the source of the imageability norms. In this study, we therefore wanted to examine potential sources of lexical and sensorimotor variance in different imageability norms. One possibility is that the variance stems from different samples of words being covered by different norming studies. For example, if a particular set of imageability norms contained more nouns, more low-frequency words, or more of some other kind of word characteristic that is likely to elicit stronger imageability effects, then it may cause discrepancies in its ability to explain word recognition relative to other norms. Alternatively, it may be due to participant variability. Although each set of norms used the same instructions and scale, they were collected from different participants who may have used different sources of information as the basis of their imageability ratings. For example, if many participants in a particular imageability norming study based their ratings of imageability on a sense of familiarity with the word (or some other proxy that relates to lexical variables included in the Study 1 null model), or on the sensorimotor grounding of the word (e.g., the visual strength of the referent concept) rather than on the actual ease of 
generating a mental image, then it may cause discrepancies in its ability to explain word recognition relative to other norms.

To identify which aspects of lexical and sensorimotor experience might contribute to differences between imageability ratings, we conducted a Principal Component Analysis (PCA) on a large number of lexical and sensorimotor variables in order to derive a core set of orthogonal lexicosemantic components, and then regressed imageability ratings from each set of norms on these components. If the norms differ greatly in how much of their variance can be explained by these lexico-semantic components, and in which components contribute most strongly, then it may offer an explanation as to why imageability ratings produced such discrepant effects on word recognition in Study 1.

\section{Method}

\section{Materials}

We used the same 9789 words (a combination of words included in the BLP and ELP analyses), lexical variables, and imageability norms from Study 1 and collated further lexical and sensorimotor variables for entering into the PCA. All 24 PCA variables are listed in Table 5. We included the zipf frequency, prevalence and familiarity variables, in addition to the standard log frequency and contextual diversity variables from ELP, to capture different aspects of word frequency and different dialects of English (i.e., UK vs US). We also included a distributional neighbourhood measure that we term linguistic distributional distance (LDD20), which was generated for the purpose of this analysis ${ }^{2}$ to maximise coverage, and was similar to Hoffman et al.'s (2013) and Shaoul and Westbury's (2010) measures of distributional neighbourhood density (i.e., diversity of words that tend to occur in a similar context to the target word), both of which were observed to affect word recognition. We included a number of wordform variables, including word length in both number of letters and syllables, and orthographic and phonological neighbourhoods. In addition, we created a

\footnotetext{
${ }^{2}$ The distributional neighbourhood of each word was created using log-transformed co-occurrence frequencies within a window of 5 words around each target word across a 200-million word subtitle corpus (see Wingfield \& Connell, 2021, for details), where LDD20 was calculated as the mean cosine distance from the vector of the target word to its 20 closest neighbours.
} 
variable to capture the noun/non-noun distinction, given the different patterns of imageability findings for nouns (e.g., Chiarello et al., 1999).

We used 11 individual dimensions of sensorimotor strength from the Lancaster Sensorimotor Norms (Lynott et al., 2020), where each dimension comprised a rating of the extent to which the word's referent was experienced with the specified perceptual modality or by performing an action with the specified action effector, thus representing real-word sensorimotor grounding of concepts. We also included Lynott et al.'s composite measure of sensorimotor strength, which aggregates all dimensions of sensorimotor experience in a single variable but attenuates the influence of weaker dimensions.

Finally, we also included age of acquisition as a lexico-semantic variable that has been shown to correlate with imageability (e.g., Clark \& Paivio, 2004) and also with a wide range of lexical and sensorimotor variables including word frequency, length, orthographic neighbourhood and visual strength (e.g., Connell \& Lynott, 2014b). 
Table 5: Variables used in Principal Components Analysis in Study 2.

\begin{tabular}{|c|c|c|c|}
\hline Original variable & Source & Definition & $\mathrm{N}$ \\
\hline LgSUBTLWF & $\overline{E L P}$ & Log word frequency (US English) & 9710 \\
\hline LgSUBTLCD & ELP & $\begin{array}{l}\text { Log contextual diversity (how many contexts } \\
\text { a word appears in: US English) }\end{array}$ & 9710 \\
\hline Zipf Frequency & Van Heuven et al. (2014) & Word frequency on Zipf scale (UK English) & 9468 \\
\hline Prevalence & Brysbaert et al. (2018) & $\begin{array}{l}\text { How many people know the word (probit } \\
\text { value) }\end{array}$ & 9468 \\
\hline Familiarity & $\begin{array}{l}\text { Stadthagen-Gonzales \& } \\
\text { Davis (2006); Scott et al. } \\
\text { (2018); Wilson (1988) }\end{array}$ & $\begin{array}{l}\text { How subjectively familiar a word seems } \\
\text { (ratings) }\end{array}$ & 6959 \\
\hline Age of Acquisition & Kuperman et al. $(2012)^{\mathrm{a}}$ & Approximate age that the word was learned & 9513 \\
\hline $\begin{array}{l}\text { Linguistic distributional } \\
\text { distance (LDD20) }\end{array}$ & $\begin{array}{l}\text { Generated for the purpose } \\
\text { of the current analysis }\end{array}$ & $\begin{array}{l}\text { Distributional neighbourhood (mean cosine } \\
\text { distance to closest } 20 \text { neighbours, based on } \\
\text { vectors of log co-occurrence frequency) }\end{array}$ & 9735 \\
\hline Word length & ELP & Word length in letters & 9786 \\
\hline Number of syllables & ELP & Word length in syllables & 9786 \\
\hline $\begin{array}{l}\text { Orthographic } \\
\text { Levenshtein Distance } \\
\text { (OLD20) }\end{array}$ & ELP & $\begin{array}{l}\text { Orthographic neighbourhood (mean letter } \\
\text { Levenshtein distance to closest } 20 \text { neighbours) }\end{array}$ & 9544 \\
\hline $\begin{array}{l}\text { Phonological } \\
\text { Levenshtein Distance } \\
\text { (PLD20) }\end{array}$ & ELP & $\begin{array}{l}\text { Phonological neighbourhood (mean phoneme } \\
\text { Levenshtein distance to closest } 20 \text { neighbours) }\end{array}$ & 9544 \\
\hline Noun (part of speech) & ELP & $\begin{array}{l}\text { Whether or not word is a noun (binary coded: } \\
\text { noun=1, non-noun=0) }\end{array}$ & 9780 \\
\hline Torso action strength & LSN & Motor strength in torso effector & 8894 \\
\hline Foot/leg action strength & LSN & Motor strength in foot/leg effector & 8894 \\
\hline Hand/arm action strength & LSN & Motor strength in hand/arm effector & 8894 \\
\hline $\begin{array}{l}\text { Composite sensorimotor } \\
\text { strength }\end{array}$ & LSN & $\begin{array}{l}\text { Aggregated sensorimotor strength in all } \\
\text { dimensions (Minkowski-3 distance of 11- } \\
\text { dimension vector from the origin) }\end{array}$ & 8894 \\
\hline Head action strength & LSN & Motor strength in head effector & 8894 \\
\hline Auditory strength & LSN & Perceptual strength in hearing modality & 8894 \\
\hline Mouth action strength & LSN & Motor strength in mouth effector & 8894 \\
\hline Gustatory strength & LSN & Perceptual strength in taste modality & 8894 \\
\hline Olfactory strength & LSN & Perceptual strength in smell modality & 8894 \\
\hline Visual strength & LSN & Perceptual strength in sight modality & 8894 \\
\hline Haptic strength & LSN & Perceptual strength in touch modality & 8894 \\
\hline Interoceptive strength & LSN & $\begin{array}{l}\text { Perceptual strength in interoceptive } \\
\text { (sensations inside the body) modality }\end{array}$ & 8894 \\
\hline
\end{tabular}

${ }^{a}$ With extended norms from http://crr.ugent.be/archives/806

Note ELP = English Lexicon project (Balota et al., 2007); LSN = Lancaster Sensorimotor Norms (Lynott et al., 2020). 


\section{Design and Analysis}

We first conducted a PCA of the 24 lexical and sensorimotor variables in order to discover how the variables cluster into larger components that represent core lexico-semantic information. We used JASP (JASP Team, 2020) to conduct a parallel analysis ( $95^{\text {th }}$ percentile), with orthogonal varimax rotation and pairwise exclusion, using the correlation matrix. This reduced the original 24 variables to an optimal 6 principal components that captured $77.4 \%$ of the original variance, with a loading value of each variable per component. We then used the Principal function (from Psych package, Revelle, 2021) ${ }^{3}$ in R to calculate and save rotated component scores for each word (a facility not currently available in JASP). We obtained 6 orthogonal components with intercorrelations of zero or near-zero. We also calculated the mean value per component for each set of imageability norms, in order to examine how the words covered by the different norms varied in lexical and sensorimotor characteristics.

We used the components in a Bayesian linear regression analysis (JASP Team, 2020; using JZS default priors, $r=.354$, beta binomial distribution $\mathrm{a}=1$ and $\mathrm{b}=1$ ) to see how much the component scores contributed to the variance in imageability ratings. We entered all 6 variables as predictors and imageability as a DV in a separate regression analysis per set of norms. Finally, we ran NHST linear regression analyses using the same structure as the Bayesian linear regression. We report effect sizes calculated from squared part correlations from the NHST regressions, and the following results from Bayesian regressions: $\mathrm{BF}_{\text {inclusion }}$ per component (i.e., indicating the relative likelihood of models including a particular component as predictor compared to models excluding it) and $\mathrm{R}^{2}$ from the most complex model from each analysis (i.e., with all components included).

\section{Results and Discussion}

The PCA resulted in six rotated components, which reflected different lexical and sensorimotor characteristics of the words. We assigned names to the components based on what types of variables loaded on them, and what type of words were representative of the components. Two

\footnotetext{
${ }^{3}$ Which is the same function used to perform PCA in JASP
} 
components represented lexical word features $(\mathrm{RC} 1=$ Frequency, $\mathrm{RC} 2=$ Length $)$ and four components represented sensorimotor experience of the referent concept $(\mathrm{RC} 3=\mathrm{Body}, \mathrm{RC} 4=$ Food, RC5 = Objects, RC6 = Communication). Variables contributing to each component are presented in Figure 2, and sample words which scored high and low on each component are presented in Table 6. 


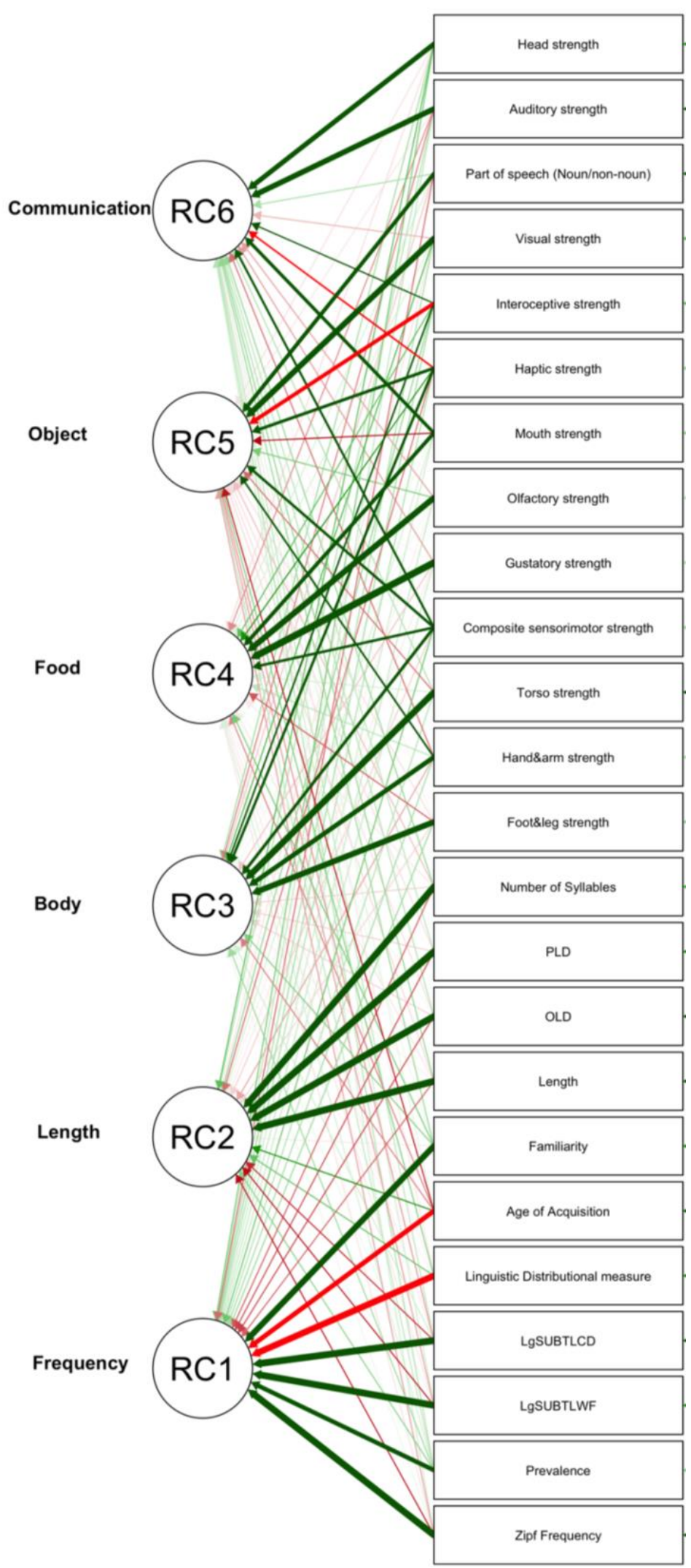

Figure 2: Rotated components obtained through the PCA, with the variables that loaded positively (green arrows) and negatively (red arrows) on each component. Thickness of line denotes strength of loading. 
Table 6: Top five (highest scoring) and bottom five (lowest scoring) words for each PCA component in Study 2.

\begin{tabular}{lll}
\hline Component & Highest scoring & Lowest scoring \\
\hline Frequency (RC1) & the, a, that, and, it & dryad, betel, quire, marl, coif \\
Length (RC2) & $\begin{array}{l}\text { Presbyterianism, Czechoslovakia, intercontinental, } \\
\text { humanitarianism, congregationalist }\end{array}$ & gab, mar, hum, sop, boll \\
Body (RC3) & diving, itchy, exercise, move, movement & an, than, so, a, the \\
Food (RC4) & meal, pizza, pastry, omelette, pasta & waltz, listen, chase, polka, ballet \\
Object (RC5) & television, keyboard, typewriter, computer, nail & $\begin{array}{l}\text { hungry, nauseous, digestion, } \\
\text { digest, gastric }\end{array}$ \\
Communication (RC6) & song, concert, joke, word, chat & $\begin{array}{l}\text { actually, molecular, than, } \\
\text { enzyme, ply }\end{array}$ \\
\hline
\end{tabular}

Mean component scores per set of norms are shown in Table 7. Overall, each set of imageability norms varied markedly in how their items relate to each component, but there were no systematic differences which could explain why imageability norms were not consistent in predicting word recognition in Study 1. For example, while words in the Bristol and Glasgow norms scored highest on the Frequency component, which could explain why imageability was not as useful when making a lexical decision in Study 1, words in the Bird norms were also high frequency, yet the Bird norms performed much better as a predictor of lexical decision and word naming. On the other hand, the Chiarello norms included the lowest frequency words, but did not elicit a consistent facilitation effect on word recognition. This suggests that the differences in words included in each set of imageability ratings are unrelated to their ability to predict word recognition, and the imageability ratings themselves vary too much across different groups of participants to consider imageability a reliable construct. 
Table 7: Mean scores per component in Study 2 (with standard deviations in parentheses) for the words covered by each set of imageability norms.

\begin{tabular}{lcccccc}
\hline Component & Bird & Bristol & Chiarello & Cortese & Glasgow & MRC \\
\hline Frequency (RC1) & $0.28(0.97)$ & $0.22(0.75)$ & $0.10(0.83)$ & $0.13(0.94)$ & $0.33(0.82)$ & $0.20(1.10)$ \\
Length (RC2) & $0.15(1.12)$ & $-0.42(0.51)$ & $-0.57(0.49)$ & $-0.48(0.61)$ & $0.07(0.97)$ & $0.01(1.05)$ \\
Body (RC3) & $0.01(1.05)$ & $0.08(1.07)$ & $0.02(1.00)$ & $-0.01(1.04)$ & $0.09(1.06)$ & $0.00(1.03)$ \\
Food (RC4) & $-0.06(0.81)$ & $-0.03(1.05)$ & $0.06(1.20)$ & $0.02(1.09)$ & $0.06(1.10)$ & $0.02(1.08)$ \\
Object (RC5) & $-0.08(0.99)$ & $-0.01(1.03)$ & $-0.01(1.04)$ & $0.02(1.03)$ & $0.12(1.05)$ & $0.11(1.02)$ \\
Communication (RC6) & $0.14(1.02)$ & $-0.11(1.02)$ & $0.09(1.14)$ & $-0.07(1.04)$ & $0.11(1.03)$ & $0.03(1.04)$ \\
\hline
\end{tabular}

In the regression analyses, the combined effect of the components on imageability ratings varied across the norms (see Figure 3), predicting $60.3 \%$ of variance in the Glasgow norms but only $38.6 \%$ of variance in the Bird norms, and the other norms ranged around $50 \%$ of variance. This difference suggests that imageability ratings from different sources differ in what they actually measure. While the components did not capture the contents of the Bird ratings well, they captured at least half the variance in all other norms, indicating that most of the imageability ratings can be predicted by lexical and sensorimotor variables to a large extent. Indeed, these results indicate that, typically, only a minority of the variance in imageability ratings can be attributed to the ease of consciously generating a mental image. Notably, the imageability norms that were least well explained by lexico-semantic components (Bird norms) performed relatively strongly in predicting word recognition in Study 1, whereas the norms that were most well explained by our components (Glasgow norms) were amongst the weakest performers in Study 1. 

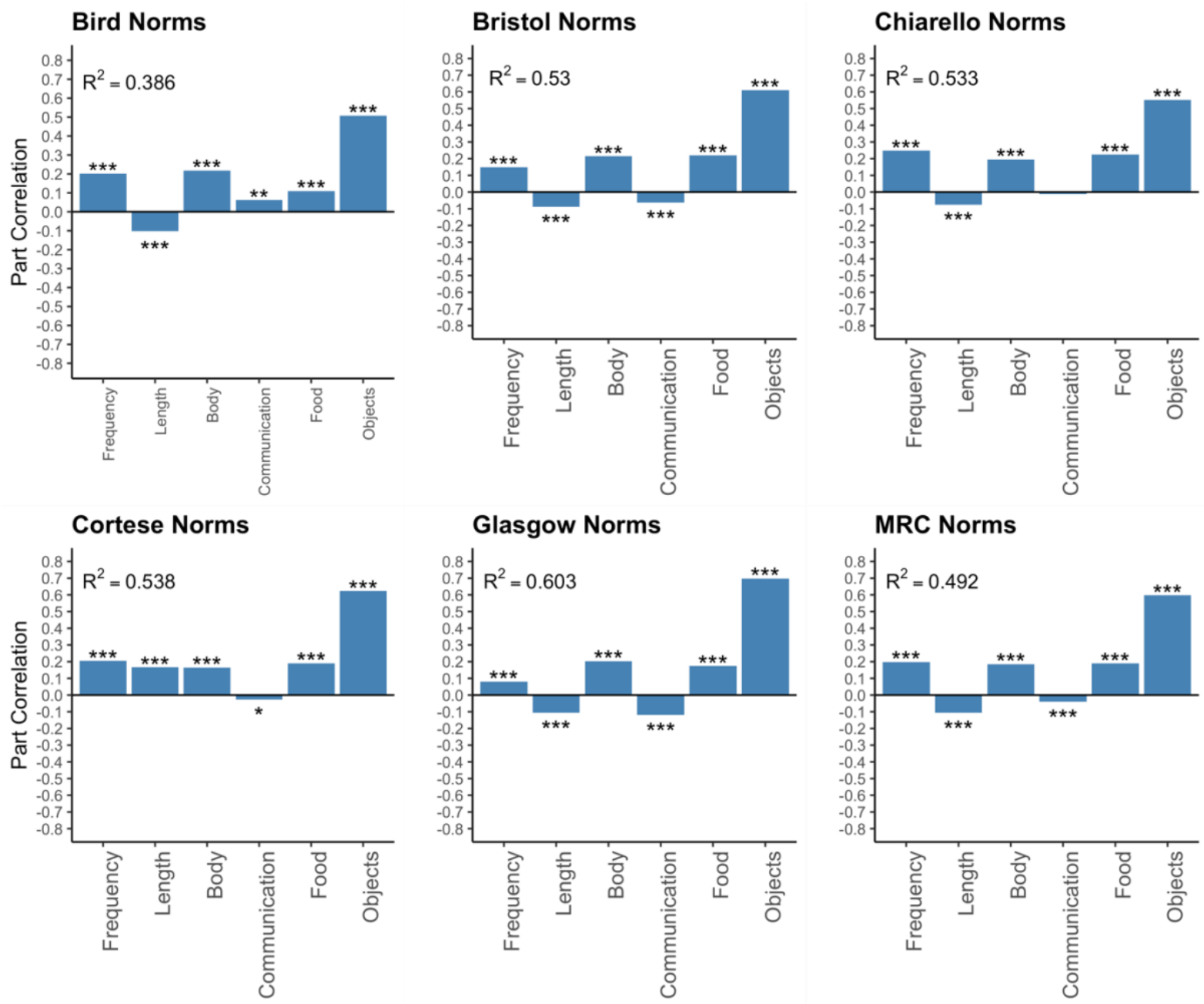

Figure 3: Part correlations of each component as a predictor of imageability ratings in Study 2 regressions per set of norms. Asterisks refer to the inclusion Bayes Factor (BF) of the coefficient: $* B F_{\text {inclusion }} \geq 3$, positive evidence; $* * \mathrm{BF}_{\text {inclusion }} \geq 20$, strong evidence; $* * * \mathrm{BF}_{\text {inclusion }} \geq 150$, very strong evidence. $R^{2}$ represents total model fit.

Based on BFs, most components contributed to all sets of imageability norms, but the effect sizes (obtained via part correlation) varied across norms and components. All norms were most strongly predicted by the Object component: that is, words were rated higher in imageability when they related strongly to external object concepts (i.e., referents of nouns which are grounded in vision, touch, and hand/arm action; and cannot be perceived via interoception). However, the effect sizes for the Object component varied substantially between $25.8 \%-48.7 \%$, depending on the imageability dataset, and largely followed the same pattern as the overall model fit (i.e., the largest Object effect size was for the Glasgow norms, the smallest for the Bird norms). Imageability ratings were also 
consistently predicted (although with varying contribution) by the Frequency $(0.7 \%-6.1 \%$,), Body $(2.7 \%-4.7 \%)$, and Food $(1.2 \%-5.0 \%)$ components: words were rated higher in imageability across all norms when they were more Frequent (i.e., highly frequent, prevalent and familiar; acquired earlier; and with denser distributional neighbourhoods), stronger in Body experience (i.e., grounded in action of the torso, foot/leg, and hand/arm; and to a lesser extent haptic and interoceptive perception) and related to Food and eating (i.e., strongly grounded in smell, taste and mouth action).

However, there were inconsistencies in the effects of the remaining components on imageability ratings. The Length component had a positive effect on the Cortese norms, whereby longer words (i.e., those with more letters and syllables; and with sparser orthographic and phonological neighbourhoods) were more likely to be rated higher in imageability, but this effect was in contrast to all the other norms where Length elicited a negative effect on imageability ratings (i.e., longer words $=$ lower imageability ratings). Words in the Cortese norms were not the shortest out of all word samples examined (see Table 7), which indicates that the inconsistent imageability effects found in Study 1 are not due to the characteristics of words used in the Cortese norms. Rather, they could be attributed to participants' tendency to lean towards rating longer words as higher in imageability in that particular set of norms. Further, the Communication component was the most inconsistent as a predictor, where words that were more strongly related to Communication (i.e., grounded in hearing and action of the mouth and head; but not perceived through touch) produced a small negative effect in most norms (Bristol, Cortese, Glasgow and MRC), but a positive effect in the Bird norms, and no effect in the Chiarello norms. Despite high component scores of the Bird, Chiarello and Cortese norms, the Communication component elicited wildly different effects on all three of these sets of imageability ratings, which again suggests that participants who rated imageability in the Bird norms were inclined to rely on a specific part of the associated simulation (i.e., auditory experience, and head and mouth action), which was not necessarily important for participants in other norming studies. This difference might explain the relatively good performance of the Bird norms in Study 1, compared to other imageability ratings. 


\section{Conclusion}

In Study 2 we identified several lexical and sensorimotor sources of variance between different imageability norms, which could explain why imageability was not a consistently reliable predictor in Study 1. In summary, results showed that imageability ratings can be predicted by whether a word referent is a manipulable object (or another noun concept that is easy to interact with manually, such as an animal), and to some extent, whether it is a frequent word which relates to food and/or bodily experience. However, imageability does not consistently reflect whether the word is short, or whether its referent is linked to communication experience. The pattern of these effects suggests that the differences between imageability norms in their ability to predict word recognition emerge from participant variability in how they consider lexico-semantic information when rating ease of generating a mental image of a word.

These findings are broadly consistent with previous work showing that when rating imageability, participants tend to rely heavily on vision (i.e., which loads on the Object component) but misinterpret sound information (i.e., which loads on the present Communication component; Connell \& Lynott, 2012a). That is, participants find it difficult to consciously consider the full range of perceptual modalities when asked to provide a single rating (Connell \& Lynott, 2016a), which may explain why imageability ratings are dominated by Object experience but inconsistently reflect Communication experience.

The extent to which words vary in sensorimotor grounding, and participants vary in what information they consider when rating imageability, could explain the differences in results of Study 1, where imageability did not reliably predict word recognition. It is possible that imageability effects are inconsistent because the ratings differ in how much sensorimotor grounding they capture. When sensorimotor information is accounted for, imageability ratings should only reflect "pure" imageability - that is, information about the ease of consciously generating mental imagery - and therefore perform more consistently when predicting word recognition. On the other hand, if participants vary in their ability to rate ease of generating mental imagery, then removing variance associated with sensorimotor grounding would not improve the ability of imageability to predict word 
recognition performance, because the ratings do not reflect any useful construct in the first place. We examine this possibility in the following studies.

\section{Study 3 - Does sensorimotor grounding facilitate word recognition?}

As demonstrated in Study 2, much of the variance in imageability can be predicted by sensorimotor grounding depending on the source of the norms. The differences in how each set of ratings relates to the sensorimotor norms is likely due to variation in how participants consider sensorimotor information associated with the words while rating imageability. However, if sensorimotor grounding of the word is accounted for, the effect that imageability ratings exert on word recognition should purely reflect the ease of generating a mental image of the concept, and should be more consistent across different norms. The orthogonalization of components in Study 2 allows us to disentangle the contributions of multiple forms of lexical and sensorimotor information, so in this study, we examine the role of sensorimotor grounding in word recognition by using the components as predictors. The contribution of sensorimotor information over and above lexical variables will shed more light on how much of word recognition can be explained by automaticallyactivated sensorimotor information (e.g., Connell \& Lynott, 2012a, 2014b; Juhasz et al., 2011), particularly in interaction with frequency (e.g., Connell \& Lynott, 2016a). We expected that the sensorimotor components would facilitate word recognition, leading to higher accuracy and faster RT.

\section{Method}

\section{Materials}

We used the same items and their 6 component scores from Study 2, and word recognition (lexical decision and word naming) data from Study 1. As before, data from the BLP dataset covered 7550 words, and the ELP dataset covered 9554 words.

\section{Design and Analysis}

We conducted hierarchical linear regressions with the six lexical and sensorimotor components as predictors, and the lexical decision accuracy and zRT (from BLP and ELP) and word 
naming accuracy and zRT (from ELP) as dependent variables. In Step 1 (null model) we entered the lexical components (Frequency and Length), in Step 2 we entered the 4 sensorimotor components (Body, Communication, Food, Object), and in Step 3 we entered the interaction of each sensorimotor component with the Frequency component. As per Study 1, there were 36 regression models in total, representing six different subsets of words (i.e., as covered by the six imageability norms) by six different dependent variables (BLP LDT RT, BLP LDT accuracy, ELP LDT RT, ELP LDT accuracy, ELP Naming RT, ELP Naming accuracy).

We first ran Bayesian linear regressions in JASP with the same (default) priors as in Study 1, from which we report Bayes Factors for model comparisons between hierarchical steps and inclusion Bayes Factors of coefficients (i.e., relative likelihood of models including a particular predictor compared to models excluding it). As in previous studies, to calculate part correlation coefficients for each predictor (i.e., the unique contribution each predictor makes to the dependent measure in question), we ran NHST linear regression analyses using the same structure as the Bayesian linear regression.

\section{Results and Discussion}

Change in $\mathrm{R}^{2}$ between hierarchical steps, and part-correlation coefficients for each component, are presented in Tables 8 and 9, and Figures 4 and 5 per word subset and task.

\section{Lexical components}

Depending on the subset of words covered by each set of norms, variables related to word frequency and length explained up to $51.2 \%$ of accuracy and $65.1 \%$ of RT in lexical decision (see Tables 8 and 9). The effects were smaller for word naming, similar to Study 1, explaining up to $24.3 \%$ of accuracy, and between $21.5 \%$ and $51.1 \%$ of word naming RT. For lexical decision, the models performed similarly for both BLP and ELP data, with accuracy having a wider range (29.9\% $51.2 \%)$ than RT $(52.7 \%-65.1 \%)$. 


\section{Sensorimotor components}

In general, Bayesian evidence for the Step 2 model (see Tables 8 and 9) suggested that the sensorimotor components facilitated accuracy and speed of word recognition. As shown by the $R^{2}$ change values in Tables 8 and 9 , the sensorimotor components collectively explained up to $3.0 \%$ of variance in word recognition over and above lexical components. However, the contribution of different types of sensorimotor information varied.

The Body component elicited the most consistent effects on lexical decision performance, explaining up to $1.0 \%$ of unique variance (based on part correlations), with some exceptions in accuracy results: the Chiarello word subset where the effect was not supported by Bayesian evidence $\left(\mathrm{BF}_{\text {inclusion }}<3\right)$, the MRC subset where the effect on BLP lexical decision was opposite to what was expected (and was very small, $<0.1 \%$, although supported by Bayesian evidence), and Bristol word subset where in Bayesian terms the effect did not appear for ELP lexical decision (although there was equivocal evidence for variable inclusion - see detailed analysis in supplemental materials). In line with previous research on semantic effects, the Body component's effects on word naming were smaller than on lexical decision (see Tables 8 and 9), with Bayesian evidence supporting the effects on word naming $\left(\mathrm{BF}_{\text {inclusion }} \geq 3\right.$ only on the largest word samples: Cortese on accuracy and $\mathrm{RT}$, and MRC on RT.

The Communication component also produced strong, although more variable facilitation effects on lexical decision, explaining up to $1.0 \%$ of variance in performance. Stronger Communication experience predicted better lexical decision performance for more than half of the word subsets $\left(\mathrm{BF}_{\text {inclusion }} \geq 3\right)$. However, there was no facilitation effect of the Communication component on word naming performance, and in fact words covered by the MRC norms were less likely to be named accurately when they were more strongly associated with auditory, interoceptive or mouth experience. 
In a similar pattern, although with lower consistency, the Food component elicited a facilitation effect $\left(\mathrm{BF}_{\text {inclusion }} \geq 3\right)$ on lexical decision $\mathrm{RT}$ about half of the time, explaining up to $0.4 \%$ of variance, but produced no effect on word naming performance, or lexical decision accuracy.

Finally, the Object component explained up to $0.2 \%$ in word recognition accuracy, and $0.4 \%$ in word recognition RT. The Object component consistently facilitated word recognition accuracy for the words covered by the Cortese and MRC norms, but unexpectedly, object-related experience impaired accuracy on BLP lexical decision and ELP word naming for the words covered by the Bird norms, and on ELP lexical decision for the words covered by the Glasgow norms. Similarly, the Object component facilitated RT on lexical decision for more than half of the word subsets, and word naming RT for two of the word subsets, but unexpectedly, it impaired BLP lexical decision accuracy and RT for words covered by the Bird norms, as well as ELP lexical decision RT for words covered by the Chiarello norms.

\section{Sensorimotor components * Frequency interaction}

When considering the interaction of the sensorimotor components with the Frequency component, the effects were more inconsistent than the Step 2 effects of the sensorimotor components. Overall, sensorimotor components*Frequency interaction explained up to $1.4 \%$ of variance.

The Body component facilitated performance on lexical decision accuracy for low frequency words which were covered by the Glasgow norms, but for words covered by the MRC norms, performance was better for high frequency words. The Body component did not interact with RT; that is, the Body component facilitated RT regardless of word frequency.

The Communication component elicited stronger facilitation effects for lower-frequency words on lexical decision for the words covered by the Chiarello norms (BLP and ELP) and the words covered by the Bird and Glasgow norms (ELP). Again, words covered by the MRC norms were recognised more accurately in BLP lexical decision and ELP word naming if they were higher in frequency, contrary to our expectations. The only interaction effect on RT was that words covered by 
the Chiarello norms were recognised faster if they were higher frequency words, otherwise the Communication component did not interact with Frequency.

There was no interaction between the Food component and the Frequency component for any of the tasks $\left(\mathrm{BF}_{\text {inclusion }}<3\right)$. The Object component elicited more accurate and faster performance on all tasks for high frequency words covered by the Bird, Cortese and MRC norms, and this pattern was also found for words covered by the Glasgow norms on ELP lexical decision. 

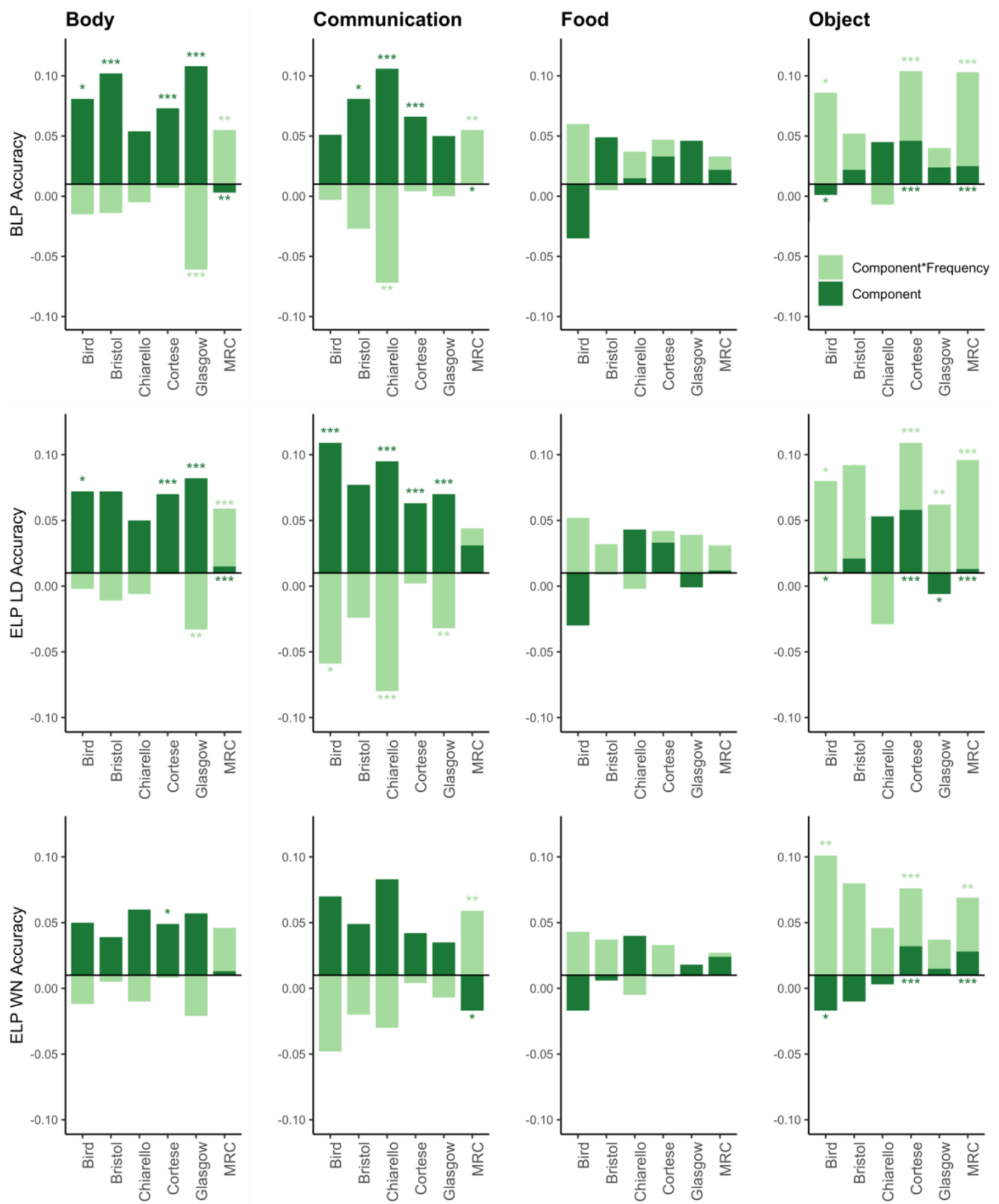

Figure 4: Part correlations of each sensorimotor component as a predictor of word recognition accuracy in the Step 3 (final) regression model of Study 3. Stacked bars represent the unique effect of sensorimotor components (darker shade) and the sensorimotor component*Frequency component interaction (lighter shade). Asterisks refer to the Bayes Factor (BF inclusion from Step 3) of the relevant parameter: $* \mathrm{BF}_{\text {inclusion }} \geq 3$, positive evidence; $* * \mathrm{BF}_{\text {inclusion }} \geq 20$, strong evidence; $* * * \mathrm{BF}_{\text {inclusion }} \geq 150$, very strong evidence. 

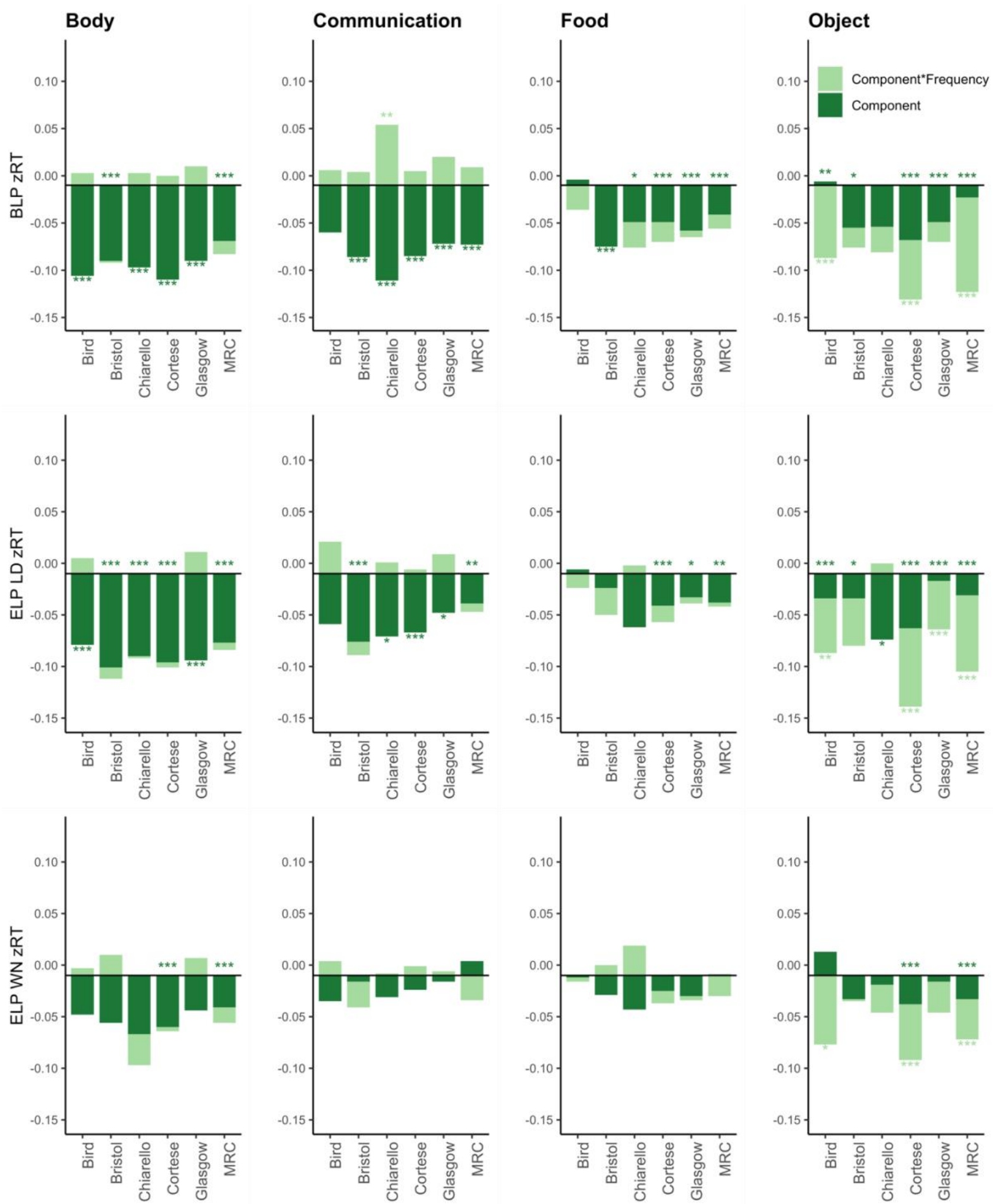

Figure 5: Part correlations of each sensorimotor component as a predictor of word recognition zRT in the Step 3 (final) regression model of Study 3. Stacked bars represent the unique effect of sensorimotor components (darker shade) and the sensorimotor component*Frequency component interaction (lighter shade). Asterisks refer to the Bayes Factor (BF inclusion from Step 3) of the relevant parameter: $* \mathrm{BF}_{\text {inclusion }} \geq 3$, positive evidence; $* * \mathrm{BF}_{\text {inclusion }} \geq 20$, strong evidence; $* * * \mathrm{BF}_{\text {inclusion }} \geq 150$, very strong evidence. 
Table 8: Variance in word recognition accuracy explained by lexical and sensorimotor components in each step of the Study 3 regression models (change in $\mathrm{R}^{2}$, with Bayes Factors for each step compared to the previous) and uniquely by each sensorimotor component and its interaction with the Frequency component in the Step 3 model (squared part correlations), for each set of imageability norms.

\begin{tabular}{|c|c|c|c|c|c|c|c|}
\hline Step & Parameter(s) & Bird & Bristol & Chiarello & Cortese & Glasgow & MRC \\
\hline \multicolumn{8}{|c|}{ BLP Lexical Decision } \\
\hline 1 & Lexical model $R^{2}$ & $.403 * * *$ & $.331 * * *$ & $.382 * * *$ & $.404 * * *$ & $.299 * * *$ & $.512 * * *$ \\
\hline 2 & Sensorimotor $\Delta R^{2}$ & .010 & $.014 * *$ & .008 & $.014 * * *$ & $.008 * * *$ & $.012 * * *$ \\
\hline \multirow[t]{9}{*}{3} & Sensorimotor*Frequency $\Delta R^{2}$ & .008 & .003 & .008 & $.004 * * *$ & $.006 *$ & $.014 * * *$ \\
\hline & Body $s r^{2}$ & .005 & .008 & .002 & .004 & .010 & .000 \\
\hline & Body*Frequency $s r^{2}$ & .001 & .001 & .000 & .000 & .005 & .002 \\
\hline & Communication $s r^{2}$ & .002 & .005 & .009 & .003 & .002 & .000 \\
\hline & Communication*Frequency $s r^{2}$ & .000 & .001 & .007 & .000 & .000 & .002 \\
\hline & Food $s r^{2}$ & .000 & .002 & .000 & .001 & .001 & .000 \\
\hline & Food*Frequency $s r^{2}$ & .003 & .000 & .000 & .000 & .000 & .000 \\
\hline & Object $s r^{2}$ & .002 & .000 & .001 & .001 & .000 & .000 \\
\hline & Object*Frequency $s r^{2}$ & .006 & .001 & .000 & .003 & .000 & .006 \\
\hline \multicolumn{8}{|c|}{ ELP Lexical Decision } \\
\hline 1 & Null model $R^{2}$ & $.343^{* * *}$ & $.367 * * *$ & $.364 * * *$ & $.406^{* * *}$ & $.301 * * *$ & $.438 * * *$ \\
\hline 2 & Sensorimotor $\Delta R^{2}$ & $.014 * *$ & .008 & .006 & $.014 * * *$ & $.006 * * *$ & $.008 * * *$ \\
\hline \multirow[t]{9}{*}{3} & Sensorimotor*Frequency $\Delta R^{2}$ & $.009 *$ & .007 & .011 & .003 & $.007^{* * *}$ & $.012 * * *$ \\
\hline & Body $s r^{2}$ & .004 & .004 & .002 & .004 & .005 & .000 \\
\hline & Body*Frequency $s r^{2}$ & .000 & .000 & .000 & .000 & .002 & .002 \\
\hline & Communication $s r^{2}$ & .010 & .004 & .007 & .003 & .004 & .000 \\
\hline & Communication*Frequency $s r^{2}$ & .005 & .001 & .008 & .000 & .002 & .000 \\
\hline & Food $s r^{2}$ & .002 & .000 & .001 & .001 & .000 & .000 \\
\hline & Food*Frequency $s r^{2}$ & .002 & .000 & .000 & .000 & .001 & .000 \\
\hline & Object $s r^{2}$ & .000 & .000 & .002 & .002 & .000 & .000 \\
\hline & Object*Frequency $s r^{2}$ & .005 & .005 & .002 & .003 & .003 & .007 \\
\hline \multicolumn{8}{|c|}{ ELP Word Naming } \\
\hline 1 & Null model $R^{2}$ & $.216^{* * *}$ & $.115^{* * *}$ & $.103^{* * *}$ & $.149^{* * *}$ & $.142^{* * *}$ & $.243 * * *$ \\
\hline 2 & Sensorimotor $\Delta R^{2}$ & .004 & .002 & .007 & $.005^{* * *}$ & .001 & $.005^{*}$ \\
\hline \multirow[t]{9}{*}{3} & Sensorimotor*Frequency $\Delta R^{2}$ & $.011^{*}$ & .007 & .003 & .003 & .002 & $.007 * * *$ \\
\hline & Body $s r^{2}$ & .002 & .001 & .003 & .002 & .002 & .000 \\
\hline & Body*Frequency $s r^{2}$ & .000 & .000 & .000 & .000 & .001 & .001 \\
\hline & Communication $s r^{2}$ & .004 & .002 & .005 & .001 & .001 & .001 \\
\hline & Communication*Frequency $s r^{2}$ & .003 & .001 & .002 & .000 & .000 & .002 \\
\hline & Food $s r^{2}$ & .001 & .000 & .001 & .000 & .000 & .000 \\
\hline & Food*Frequency $s r^{2}$ & .001 & .001 & .000 & .001 & .000 & .000 \\
\hline & Object $s r^{2}$ & .001 & .000 & .000 & .000 & .000 & .000 \\
\hline & Object*Frequency $s r^{2}$ & .008 & .005 & .001 & .002 & .000 & .002 \\
\hline
\end{tabular}

Note. The summed $\mathrm{s}^{2}$ for sensorimotor and sensorimotor*Frequency components in the final model can be greater than the corresponding $\Delta R^{2}$ due to mutual suppression of parameters.

$* \mathrm{BF}_{10} \geq 3$, positive evidence; ** $\mathrm{BF}_{10} \geq 20$, strong evidence; *** $\mathrm{BF}_{10} \geq 150$, very strong evidence. 
Table 9: Variance in word recognition RT explained by lexical and sensorimotor components in each step of the Study 3 regression models (change in $\mathrm{R}^{2}$, with Bayes Factors for each step compared to the previous) and uniquely by each sensorimotor component and its interaction with the Frequency component in the Step 3 model (squared part correlations), for each set of imageability norms.

\begin{tabular}{|c|c|c|c|c|c|c|c|}
\hline Step & Parameter(s) & Bird & Bristol & Chiarello & Cortese & Glasgow & MRC \\
\hline \multicolumn{8}{|c|}{ BLP Lexical Decision } \\
\hline 1 & Lexical model $R^{2}$ & $.555^{* * * *}$ & $.538 * * *$ & $.585^{* * * *}$ & $.544 * * *$ & $.557 * * *$ & $.606 * * *$ \\
\hline 2 & Sensorimotor $\Delta R^{2}$ & $.019^{* * *}$ & $.021 * * *$ & $.020 * * *$ & $.030^{* * *}$ & $.015^{* * *}$ & $.027 * * *$ \\
\hline \multirow[t]{9}{*}{3} & Sensorimotor*Frequency $\Delta R^{2}$ & .006 & .001 & .006 & $.004 * * *$ & .002 & $.011^{* * *}$ \\
\hline & Body $s r^{2}$ & .009 & .006 & .008 & .010 & .006 & .003 \\
\hline & Body*Frequency $s r^{2}$ & .000 & .000 & .000 & .000 & .000 & .000 \\
\hline & Communication $s r^{2}$ & .003 & .006 & .010 & .006 & .004 & .004 \\
\hline & Communication*Frequency $s r^{2}$ & .000 & .000 & .004 & .000 & .001 & .000 \\
\hline & Food $s r^{2}$ & .000 & .004 & .002 & .002 & .002 & .001 \\
\hline & Food*Frequency $s r^{2}$ & .001 & .000 & .001 & .000 & .000 & .000 \\
\hline & Object $s r^{2}$ & .000 & .002 & .002 & .003 & .002 & .000 \\
\hline & Object*Frequency $s r^{2}$ & .006 & .000 & .001 & .004 & .000 & .010 \\
\hline \multicolumn{8}{|c|}{ ELP Lexical Decision } \\
\hline 1 & Null model $R^{2}$ & $.641 * * *$ & $.527 * * *$ & $.558 * * *$ & $.531 * * *$ & $.607 * * *$ & $.651 * * *$ \\
\hline 2 & Sensorimotor $\Delta R^{2}$ & $.012 * * *$ & $.020^{* * *}$ & $.015^{* * *}$ & $.026^{* * *}$ & $.011^{* * *}$ & $.017 * * *$ \\
\hline \multirow[t]{9}{*}{3} & Sensorimotor*Frequency $\Delta R^{2}$ & .003 & .003 & .000 & $.006^{* * *}$ & $.003^{* *}$ & $.006^{* * *}$ \\
\hline & Body $s r^{2}$ & .005 & .008 & .006 & .007 & .007 & .004 \\
\hline & Body*Frequency $s r^{2}$ & .000 & .000 & .000 & .000 & .000 & .000 \\
\hline & Communication $s r^{2}$ & .002 & .004 & .004 & .003 & .001 & .001 \\
\hline & Communication*Frequency $s r^{2}$ & .001 & .000 & .000 & .000 & .000 & .000 \\
\hline & Food $s r^{2}$ & .000 & .000 & .003 & .001 & .001 & .001 \\
\hline & Food*Frequency $s r^{2}$ & .000 & .001 & .000 & .000 & .000 & .000 \\
\hline & Object $s r^{2}$ & .001 & .001 & .004 & .003 & .000 & .000 \\
\hline & Object*Frequency $s r^{2}$ & .003 & .002 & .000 & .006 & .002 & .005 \\
\hline \multicolumn{8}{|c|}{ ELP Word Naming } \\
\hline 1 & Null model $R^{2}$ & $.511^{* * *}$ & $.240^{* * *}$ & $.215^{* * *}$ & $.314 * * *$ & $.429 * * *$ & $.503 * * *$ \\
\hline 2 & Sensorimotor $\Delta R^{2}$ & .003 & .003 & .005 & $.008^{* * *}$ & .002 & $.007 * * *$ \\
\hline \multirow[t]{9}{*}{3} & Sensorimotor*Frequency $\Delta R^{2}$ & .005 & .001 & .003 & $.003 *$ & .001 & $.003^{* *}$ \\
\hline & Body $s r^{2}$ & .001 & .002 & .003 & .003 & .001 & .001 \\
\hline & Body*Frequency $s r^{2}$ & .000 & .000 & .001 & .000 & .000 & .000 \\
\hline & Communication $s r^{2}$ & .001 & .000 & .000 & .000 & .000 & .000 \\
\hline & Communication*Frequency $s r^{2}$ & .000 & .001 & .000 & .000 & .000 & .001 \\
\hline & Food $s r^{2}$ & .000 & .000 & .001 & .000 & .000 & .000 \\
\hline & Food*Frequency $s r^{2}$ & .000 & .000 & .001 & .000 & .000 & .000 \\
\hline & Object $s r^{2}$ & .001 & .001 & .000 & .001 & .000 & .001 \\
\hline & Object*Frequency $s r^{2}$ & .004 & .000 & .001 & .003 & .001 & .002 \\
\hline
\end{tabular}

Note. The summed $\mathrm{s}^{2}$ for sensorimotor and sensorimotor*Frequency components in the final model can be greater than the corresponding $\Delta R^{2}$ due to mutual suppression of parameters.

$* \mathrm{BF}_{10} \geq 3$, positive evidence; ** $\mathrm{BF}_{10} \geq 20$, strong evidence; *** $\mathrm{BF}_{10} \geq 150$, very strong evidence. 


\section{Conclusion}

Overall, word subsets covered by different norms varied in how well their recognition was predicted by sensorimotor grounding. This variability was not entirely unexpected, because the word subsets clearly differed in their sensorimotor characteristics (see Study 2, Table 7), but no particular pattern emerged which could explain these discrepancies. Thus, a set of words strongly grounded in, for example, Object experience (e.g., as covered by the Glasgow and MRC norms), can vary in how well their recognition performance is predicted by that component. Therefore, the differences in performance between tasks and measures cannot be explained by sensorimotor grounding. Instead, it appears that different words vary in how their grounding affects participant behaviour and judgement in word recognition tasks.

It is important to note that each component, which captured different aspects of sensorimotor experience, facilitated word processing to a varying degree. Additionally, recognition of some of the word subsets was actually impaired by some aspects of sensorimotor experience (e.g., stronger Object scores were associated with worse lexical decision accuracy in the sample of words covered by the Bird norms), although again there was no clear pattern to this effect. These findings suggest that the role of sensorimotor information in word recognition is more complex than originally assumed (Connell \& Lynott, 2012a; Juhasz et al., 2011; Yap et al., 2011), and warrants its own investigation. We address this point in more detail in the general discussion.

Study 3 has established the extent to which lexical characteristics of words and sensorimotor grounding of the referent concepts can facilitate word recognition, meaning that the residuals of this analysis represent the remaining variance in word recognition performance once these effects have been removed. In the next Study 4, we revisit our initial research question of whether imageability is a stable predictor of performance in lexical decision and word naming tasks by analysing its ability to predict the residuals of lexical and sensorimotor variables. Any imageability effect that emerges would thus be a "pure" imageability contribution which reflects only participants' ease of generating a mental image, independent of other sources of variance in imageability ratings such as sensorimotor grounding. 


\section{Study 4 - What is the "pure" effect of imageability on word recognition?}

Given the degree to which sensorimotor components predict imageability (Study 2), and the effects of sensorimotor grounding on word recognition (Study 3), it is important to determine whether imageability elicits an effect independently of sensorimotor grounding. Previous work has found mixed effects. While Connell and Lynott (2012a) found that imageability from the MRC norms had no independent effect above sensorimotor strength (see also Speed \& Brysbaert, 2021, for similar results in Dutch), Khanna and Cortese (2021) found that imageability from the Cortese norms still elicited an effect on word recognition when sensorimotor grounding was accounted for. Khanna and Cortese concluded the differences in findings may be due to using different variables in the baseline models (particularly AoA), but other, potentially complementary, explanations include analysing different samples of words and using different sources of imageability norms, both of which can produce large differences in imageability effects (i.e., Study 1 in the present paper). Moreover, while these previous studies used a single composite variable of perceptual and/or action strength (e.g., maximum rating across multiple dimensions), in Study 3 we found that sensorimotor strength effects varied in magnitude depending on the types of sensorimotor experience analysed (i.e., relating to Body, Communication, Food, Objects), which suggests that a single composite variable may not be the most effective way to examine sensorimotor grounding. Analysing the contribution of sensorimotor grounding across multiple orthogonal components therefore gives a more nuanced picture of the role of sensorimotor grounding in word recognition.

In Study 4 we therefore examine whether ease of generating a mental image reliably facilitates word recognition after the contribution of lexical and sensorimotor variance is removed by regressing the residuals ${ }^{4}$ of a lexico-sensorimotor model on imageability ratings from a range of different sources. Specifically, we remove effects of the high-performing lexical model from Study 1

\footnotetext{
${ }^{4}$ We opted not to employ a simple hierarchical model in this study (i.e., adding imageability and the imageability*frequency interaction in subsequent steps) because the strong but variable relationships between imageability and sensorimotor components that we observed in Study 2 meant that suppression effects were likely to distort estimates of effect size and make it difficult to compare effects across sets of norms.
} 
and sensorimotor grounding across multiple discrete components (i.e., Body, Communication, Food, Objects) as per Study 3, and only then determine what remaining variance can be explained by imageability and its interaction with frequency. Any effect of imageability that emerges in this study can therefore be considered a "pure" effect that reflects the ease of consciously generating a mental image for a word (i.e., its intended construct), and nothing else.

\section{Method}

\section{Materials}

We used the sensorimotor components from Study 3, with the 6 different sets of imageability norms and corresponding lexical variables from Study 1. As in Study 3, data from the BLP dataset covered 7550 words, and the ELP dataset covered 9554 words.

\section{Design and Analysis}

To create the dependent variables for the present study, we extracted the residuals of a baseline linear regression model containing the best-fitting lexical predictors from Study 1 (word length, number of syllables, LgSUBTLCD, OLD, prevalence $)^{5}$, the four sensorimotor components from Study 2 (Body, Communication, Food, Objects), and the interactions of each sensorimotor component*prevalence on each of the six word recognition measures from Studies 1 and 3: accuracy and RT of lexical decision from BLP and ELP and of word naming from ELP. We report the fit of these baseline models as Step 0 in regression analyses. Residuals were separately calculated for each different subset of words (i.e., as covered by the six imageability norms) using $\operatorname{lm}$ function in $\mathrm{R}$ (Venables \& Ripley, 2002).

\footnotetext{
${ }^{5}$ This high-performing lexical model from Study 1 does not include AoA, yet it performs better than the lexical components in Study 3 that did include AoA. We therefore use the Study 1 lexical model here for to maximise variance explained prior to determining the contribution of imageability, but include in supplementals an analysis where imageability is assessed on the residuals of Study 3 (i.e., where AoA is incorporated in the PCA components). Imageability effect sizes are generally slightly larger in this supplemental analysis, though qualitatively very similar to the present study.
} 
We then ran hierarchical linear regressions on these residual DVs. In Step 1 we entered imageability (centred) and in Step 2 we entered the interaction of imageability*frequency (represented by prevalence, centred for the whole dataset). As in Studies 1 and 3, there were 36 regression models in total, representing six different imageability norms (Bird, Bristol, Chiarello, Cortese, Glasgow, MRC) by the residuals of six different dependent variables (BLP LDT RT, BLP LDT accuracy, ELP LDT RT, ELP LDT accuracy, ELP Naming RT, ELP Naming accuracy).

As in previous studies, we ran Bayesian linear regressions in JASP (JASP Team, 2020) with the same (default) priors as in Study 1, from which we report Bayes Factors for model comparisons between hierarchical steps and inclusion Bayes Factors of coefficients (i.e., relative likelihood of models including a particular predictor compared to models excluding it). Again, to calculate part correlation for each predictor (i.e., the unique contribution each predictor makes to the dependent measure in question), we ran NHST linear regression analyses using the same structure as the Bayesian linear regression. To enable cross-study comparisons, we report effect sizes as variance of the original DV (i.e., accuracy or RT), which we calculated by scaling each $R^{2}$-change and squared part correlation by the size of the relevant residual variance of the baseline lexico-sensorimotor regression model.

\section{Results and Discussion}

Change in $\mathrm{R}^{2}$ between hierarchical steps, and part-correlation coefficients for imageability and its Frequency interaction, for each set of imageability norms and each task are presented in Table 10 and Figure 6. These statistics reflect variance explained in each original DV (i.e., word recognition accuracy or RT) for comparison with Study 1; full results of variance explained in the residuals is available in supplementals. 

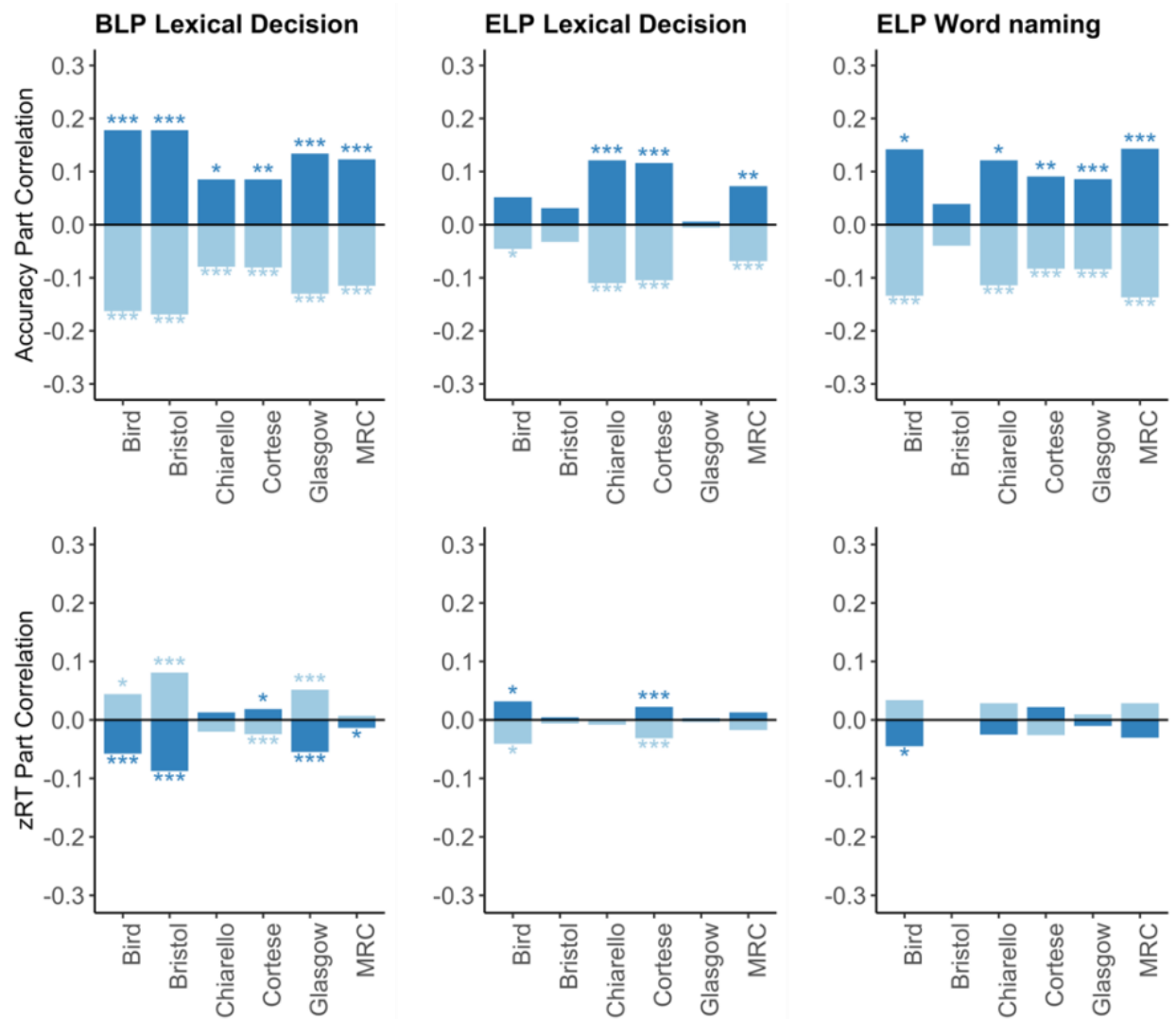

Imageability*Prevalence Imageability

Figure 6: Part correlations of imageability as a predictor of word recognition accuracy (top row) and zRT (bottom row) in the Step 2 (final) regression model of Study 4. Stacked bars represent the unique effect of imageability (darker shade) and the imageability*frequency interaction (lighter shade). Asterisks refer to the Bayes Factor (BF inclusion from Step 2 ) of the relevant parameter: $* \mathrm{BF}_{\text {inclusion }} \geq 3$, positive evidence; $* *$ $\mathrm{BF}_{\text {inclusion }} \geq 20$, strong evidence; $* * * \mathrm{BF}_{\text {inclusion }} \geq 150$, very strong evidence. 
Table 10: Variance in word recognition accuracy and RT explained by each step of the Study 4 regression models (change in $R^{2}$, with Bayes Factors for each step compared to the previous) and uniquely explained by imageability and its frequency interaction in the Step 2 model (squared part correlations) for each set of imageability norms. Effect sizes are scaled to reflect variance of the original DV rather than variance of the residuals only.

\begin{tabular}{|c|c|c|c|c|c|c|c|}
\hline Step & Parameter(s) & Bird & Bristol & Chiarello & Cortese & Glasgow & MRC \\
\hline \multicolumn{8}{|c|}{ BLP Lexical Decision accuracy } \\
\hline 0 & Baseline model $R^{2}$ & $.680^{* * *}$ & $.494 * * *$ & $.624 * * *$ & $.685^{* * *}$ & $.445^{* * *}$ & $.724 * * *$ \\
\hline 1 & Imageability $R^{2}$ & $.008^{* * * *}$ & $.006^{* * * *}$ & .002 & $.001^{* * *}$ & $.002 *$ & $.003^{* * * *}$ \\
\hline \multirow[t]{3}{*}{2} & Imageability*frequency $\Delta R^{2}$ & $.035^{* * * *}$ & $.035^{* * * *}$ & $.008^{* * *}$ & $.008^{* * *}$ & $.019^{* * *}$ & $.016^{* * * *}$ \\
\hline & Imageability $s r^{2}$ & .052 & .031 & .121 & .116 & .006 & .072 \\
\hline & Imageability*frequency $s r^{2}$ & .046 & .033 & .110 & .105 & .006 & .069 \\
\hline \multicolumn{8}{|c|}{ ELP Lexical Decision accuracy } \\
\hline 0 & Baseline model $R^{2}$ & $.656^{* * *}$ & $.575^{* * *}$ & $.585^{* * *}$ & $.672^{* * *}$ & $.519^{* * *}$ & $.685^{* * *}$ \\
\hline 1 & Imageability $R^{2}$ & .001 & .000 & $.006^{* * * *}$ & $.005^{* * *}$ & .000 & $.001 *$ \\
\hline \multirow[t]{3}{*}{2} & Imageability*frequency $\Delta R^{2}$ & $.003 *$ & .001 & $.018^{* * * *}$ & $.016^{* * *}$ & .000 & $.005^{* * *}$ \\
\hline & Imageability $s r^{2}$ & .178 & .178 & .085 & .085 & .134 & .123 \\
\hline & Imageability*frequency $s r^{2}$ & .163 & .169 & .079 & .081 & .130 & .115 \\
\hline \multicolumn{8}{|c|}{ ELP Word Naming accuracy } \\
\hline 0 & Baseline model $R^{2}$ & $.424 * * *$ & $.222^{* * * *}$ & $.228^{* * *}$ & $.284 * * *$ & $.238 * * *$ & $.415^{* * * *}$ \\
\hline 1 & Imageability $R^{2}$ & .003 & .000 & .003 & $.002 * *$ & .000 & $.002 *$ \\
\hline \multirow[t]{3}{*}{2} & Imageability*frequency $\Delta R^{2}$ & $.021^{* * * *}$ & .002 & $.016^{* * * *}$ & $.009^{* * *}$ & $.007^{* * * *}$ & $.020 * * *$ \\
\hline & Imageability $s r^{2}$ & .142 & .039 & .121 & .091 & .086 & .143 \\
\hline & Imageability*frequency $s r^{2}$ & .134 & .040 & .114 & .083 & .084 & .137 \\
\hline \multicolumn{8}{|c|}{ BLP Lexical Decision $z R T$} \\
\hline 0 & Baseline model $R^{2}$ & $.690 * * *$ & $.568^{* * * *}$ & $.650^{* * *}$ & $.662 * * *$ & $.581^{* * *}$ & $.671^{* * * *}$ \\
\hline 1 & Imageability $R^{2}$ & $.005^{* * * *}$ & $.004 * *$ & .001 & $.001 *$ & .001 & $.001 * *$ \\
\hline \multirow[t]{3}{*}{2} & Imageability*frequency $\Delta R^{2}$ & .007 & $.010^{* * *}$ & .002 & $.001 *$ & $.004^{* * *}$ & .001 \\
\hline & Imageability $s r^{2}$ & .058 & .087 & .013 & .019 & .055 & .014 \\
\hline & Imageability*frequency $s r^{2}$ & .044 & .081 & .020 & .024 & .052 & .007 \\
\hline \multicolumn{8}{|c|}{ ELP Lexical Decision $z R T$} \\
\hline 0 & Baseline model $R^{2}$ & $.709 * * *$ & $.607 * * *$ & $.619^{* * * *}$ & $.651^{* * * *}$ & $.663^{* * *}$ & $.712^{* * * *}$ \\
\hline 1 & Imageability $R^{2}$ & .001 & .000 & .001 & $.002 * * *$ & .000 & .000 \\
\hline \multirow[t]{3}{*}{2} & Imageability*frequency $\Delta R^{2}$ & $.003 *$ & .000 & .001 & $.003^{* * *}$ & .000 & .001 \\
\hline & Imageability $s r^{2}$ & .032 & .004 & .001 & .022 & .003 & .013 \\
\hline & Imageability*frequency $s r^{2}$ & .041 & .006 & .008 & .031 & .003 & .017 \\
\hline \multicolumn{8}{|c|}{ ELP Word Naming $z R T$} \\
\hline 0 & Baseline model $R^{2}$ & $.539 * * *$ & $.299 * * *$ & $.244 * * *$ & $.382^{* * * *}$ & $.459^{* * * *}$ & $.538 * * *$ \\
\hline
\end{tabular}




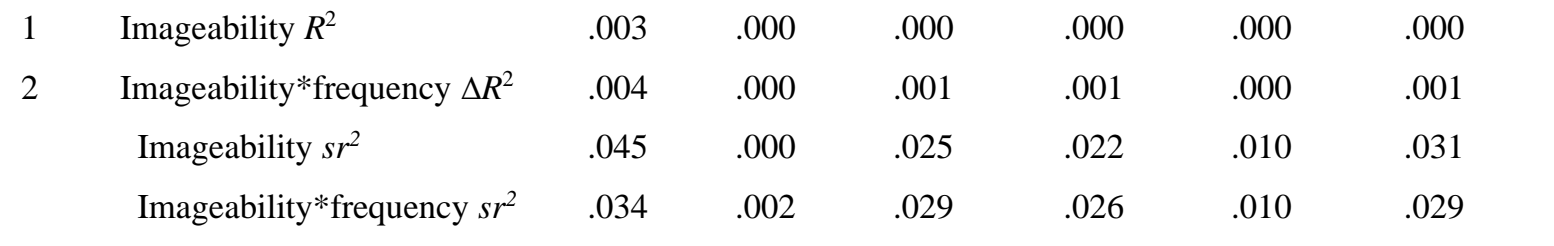

Note. The summed $\mathrm{s} r^{2}$ for imageability and imageability*frequency components in the final model can be greater than the corresponding $\Delta R^{2}$ due to mutual suppression of parameters.

$* \mathrm{BF}_{10} \geq 3$, positive evidence; $* * \mathrm{BF}_{10} \geq 20$, strong evidence; *** $\mathrm{BF}_{10} \geq 150$, very strong evidence.

\section{Imageability}

As shown by the change in $R^{2}$ for the Step 1 models in Table 10, imageability contributed up to $0.8 \%$ of the variance in word recognition over and above lexical and sensorimotor components. However, imageability had no effect at all in over half of the Step 1 analyses (21 out of 36 models), and almost all effects were markedly smaller than in Study 1. Moreover, the contribution of "pure" imageability to word recognition varied enormously between the norms and datasets.

As we found in Study 1, no set of imageability norms were consistent in facilitating accuracy and RT for all 6 DVs. The Cortese norms performed best, with effects in 5 out of 6 DVs, followed by the MRC norms at 4 out of 6 DVs, although these effects were all very small (i.e., $<0.5 \%$ of variance). The remaining sets of norms contributed intermittently to word recognition, producing imageability effects on either two DVs (Bird, Bristol) or on one a single DV (Chiarello, Glasgow). Overall, these results suggest that isolating a "pure" effect of imageability on word recognition by removing lexical and sensorimotor information does not smooth out the variability in effects that we saw in Study 1, but instead increases variability while diminishing the overall imageability effect size.

There were again systematic differences between tasks and dataset sources. Imageability effect sizes were larger for accuracy than RT, regardless of task (see standardised regression coefficients and part correlations in supplementals), unlike in Study 1 where effects on lexical decision were larger for RT than accuracy. As per Study 1, imageability effects were overall stronger (and more consistently elicited) for BLP data than ELP data, but there was no longer any systematic difference between lexical decision and word naming tasks. 


\section{Imageability*frequency interaction}

Step 2 models indicated that the imageability*frequency interaction explained up to $3.5 \%$ of variance over imageability alone. However, this effect size was an exception (Bird and Bristol norms, BLP lexical decision accuracy), and while the interaction had an effect on word recognition in half of the analyses (19 out of 36 models), its contribution was very small ( $<1 \%$ variance) in many cases, with large differences between norms and datasets.

There were interaction effects on accuracy for most sets of norms. The Bird, Chiarello, Cortese and MRC norms elicited an imageability*frequency effect on accuracy across all tasks, while the Bristol norms only elicited an effect on BLP lexical decision, and Glasgow norms on BLP lexical decision and ELP word naming. As in Study 1, no set of norms consistently produced stronger facilitation for low-frequency words for both accuracy (i.e., negative part correlation for interaction term) and RT (i.e., positive part correlation for interaction term), largely because the interaction effects were sparse and highly variable for RT. While the Bird, Bristol and Glasgow norms produced the expected interaction effects on RT for BLP lexical decision, the only two interaction effects for ELP lexical decision ran in the opposite direction to predictions (i.e., larger Bird and Cortese imageability effect for high frequency words), and no set of norms elicited an interaction effect on ELP word naming RT. Overall, interaction effects in the present study were smaller than those obtained in Study 1, but were similar in terms of the direction of these effects.

There were also systematic differences between dataset sources. In general, the imageability*frequency interaction was more likely to emerge for accuracy ( 15 out of 18 models) than for RT ( 5 out of 18 models, but only 2 of these in the expected direction), and the interaction effects were overall larger for BLP than ELP. As per Study 1, there were no consistent task differences: within the ELP, the expected interaction effects appeared more often for word naming than for lexical decision, but effects for BLP lexical decision were consistently stronger than ELP effects.

\section{Conclusion}

We compared how well six sets of imageability norms contributed to word recognition performance, using residual variance from a regression analysis where the effects due to other lexical 
and sensorimotor variables have been removed. This approach allowed us to analyse the "pure" effect of the ease of generating mental imagery, without the confounding effects of lexical characteristics and sensorimotor grounding; something that we could not fully control in Study 1.

We found that "pure" imageability can contribute to word recognition, but does not do so consistently as large differences are evident across different sources of imageability norms. None of the norms consistently predicted word recognition, although most of them elicited some effects on accuracy. The best ratings at eliciting reliable "pure" imageability effects, with mostly large effects for low frequency words, were the Bird norms, though even these ratings were prone to produce interactions opposite to predictions. The MRC norms also elicited "pure" imageability effects, though often weakly, and the Chiarello and Glasgow norms produced similar but less reliable results. On the other hand, the Cortese norms had unusual effects on lexical decision RT (i.e., imageability led to more accurate but slower responding for low-frequency words), while the Bristol norms could not detect imageability effects with any consistency. This pattern of results indicates that ease of generating mental imagery can at least sometimes elicit an effect on word recognition performance independently of sensorimotor grounding, though primarily it influences accuracy rather than latency. However, different sets of imageability ratings are not consistent and robust in capturing this "pure" measure of ease of mental imagery. Such variable effects mirror those of the literature, where imageability sometimes elicits an effect above and beyond sensorimotor information (Khanna \& Cortese, 2021; Vergallito et al., 2020, for Italian data), but other times it does not (Connell \& Lynott, 2012a; Speed \& Brysbaert, 2021; Vergallito et al., 2020, for English data; see also Sidhu et al., 2014), even when exactly the same baseline models are used. Instead, the source of imageability norms analysed is much more likely to determine whether unique effects of imageability can be detected.

When Study 2 showed that imageability norms differed in how much sensorimotor grounding they captured, we queried whether it could explain the discrepant pattern of imageability effects we found in Study 1. If this were the case, then removing the effects of sensorimotor information in the present study should have led different sets of imageability norms to perform more consistently when predicting word recognition. However, no such consistency emerged: imageability effects remained 
unstable from one set of norms to the next. This finding suggests that the variable utility of different imageability norms lies in the ratings themselves, or more specifically in the type of information that participants in imageability norming studies used as the basis of their ratings. For example, some participants might be diligent and accurate when quantifying the ease of generating a mental image, whereas others may interpret the instructions differently: they may concentrate on visual imagery to the exclusion of other sensorimotor dimensions, or rate the overall strength or breadth of sensorimotor experience in the referent concept instead of the ease of generating imagery, or allow their familiarity with the wordform itself to influence ratings. Indeed, Study 2 showed that only $39.7 \%$ of the variance in imageability ratings from the Glasgow norms comes from something other than lexical and sensorimotor information. At least some of this $39.7 \%$ variance would be expected to reflect "pure" imageability, the actual ease of generating a mental image of the word. Yet, as we saw in the present Study 4 , this additional variance was not consistently useful in predicting word recognition performance. We return to this issue in the general discussion.

In short, the variability in imageability effects indicates that, despite using the same instructions and scales in every norming study, the construct of imageability itself is not stable. There is so much variability in the kind of information participants incorporate when providing their ratings that the notion of imageability itself - that is, the ease of generating a mental image - is not a psychological construct which can be reliably rated, at least not in a way that produces reliable behavioural effects on word processing. Given this level of inconsistency, and the large number of null effects observed in the present study, it is not clear whether or not imageability should be regarded as having a general effect on word recognition performance. In order to address that question, and quantify a summary effect size, we conducted a meta-analysis of imageability effects on word recognition.

\section{Study 5 - Is there a meta-effect of imageability on word recognition?}

Due to the large variability in imageability effects between datasets in Study 4, we conducted an internal meta-analysis of the 36 imageability part correlations from Study 4 (i.e., the "pure" imageability effect after lexical and sensorimotor variability had been partialled out) with three goals 
in mind. First, we wanted to determine the degree of heterogeneity and overall meta-effect size of imageability on word recognition. If imageability effects emerged often enough, and strongly enough, in Study 4 (despite frequent null effects), then a meta-effect of imageability on word recognition should be greater than zero. Second, because some of the observed differences in effect sizes could perhaps be attributed to moderators (e.g., imageability may have a different effect on latency versus accuracy of word recognition performance, or on lexical decision versus word naming), we also examined a range of potential moderating variables. Third and final, we wanted to quantify a metaeffect size per set of norms, which would allow us to determine the overall robustness of each set of imageability norms in facilitating word recognition performance, regardless of dataset analysed.

\section{Method}

\section{Materials}

As effect sizes for analysis, we used part correlation coefficients of the imageability effects on word recognition residuals from Study 4, which reflected "pure" imageability effects independent of variance associated with lexical and sensorimotor variables, scaled to reflect contribution to the original accuracy and zRT dependent variables rather than to the residuals. There were 36 effect sizes, 6 per set of norms (effects on accuracy and RT from BLP lexical decision data and ELP lexical decision and word naming data). In order to obtain standardised effect sizes and their standard errors, we transformed $s r$ to Fisher's $\mathrm{Zr}$ and calculated its $\mathrm{SE}^{6}$. (We then transformed the $\mathrm{Zr}$ back to $s r$ when calculating and reporting effect sizes). Because accuracy and RT are expected to show opposite effect directions (better accuracy means a positive effect size, while faster RT means a negative effect size), we also transformed the standardised effect sizes to reflect a 'facilitation' effect by reversing the sign of each RT effect. Positive standardised effect sizes thus represent facilitation of word recognition (faster or more accurate), while negative standardised effect sizes represent inhibition (slower or less accurate).

\footnotetext{
${ }^{6}$ Using the formula as per Lipsey and Wilson (2001): $Z_{r}=\frac{1}{2} \times((\ln (1+r))-(\ln (1-r))) ; S E=1 / \sqrt{n-3}$, where $n=$ the number of words analysed in the sample; R code available in supplementals.
} 


\section{Design and Analysis}

We ran all meta-analyses of imageability effect sizes in JASP (2020). We used a random effects model with Restricted Maximum Likelihood, which is considered a good method to estimate between-study variance (Veroniki et al., 2015) when there is significant heterogeneity (Cumming, 2012).

Due to the large differences observed between norms, tasks and measures in Study 4, we entered a number of variables as candidate moderators in the analysis in an attempt to improve model fit and to obtain a more accurate picture of what influences reliability of imageability as a predictor of word recognition. The candidate moderators were: norms (6 sets), task type (lexical decision, word naming), DV (accuracy, zRT), source (BLP, ELP), and geographical congruence (congruent, incongruent; reflecting whether or not the country of origin of the imageability norms matched the country where the word recognition data was collected) ${ }^{7}$. We used the Bayesian Information Criterion (BIC), where lower BIC means better fit, to determine the best fitting model (see supplementals for full details) and to approximate Bayes Factors for model comparison (e.g., Wagenmakers, 2007). The best model had DV as a moderator, which was $\mathrm{BF}_{10}=32597.80$ times better than the model with no moderators, and $\mathrm{BF}_{10}=1.96$ times better than the next-best moderator model (with DV and source); we therefore selected the more parsimonious model (with DV only) as best fit.

We first report a global analysis on all data points, then a meta-analysis with DV (accuracy vs RT) as a moderator, and then a separate analysis for each set of norms (moderated by DV) to establish their independent robustness in facilitating word recognition performance.

\section{Results and Discussion}

In the global meta-analysis of all 36 imageability effects, we observed that $91 \%$ of the variance was due to heterogeneity between datasets (i.e., between different norms, tasks and

\footnotetext{
${ }^{7}$ We also conducted pairwise correlations between each set of norms to further examine whether ratings from the same country of origin may be more closely related than ratings which originate from different countries, but no systematic relationship emerged (see supplemental materials for details). We thank an anonymous reviewer for this suggestion.
} 
measures; $\left.\mathrm{I}^{2}=90.83,95 \% \mathrm{CI}=[85.76,95.71]\right)$. The overall meta-effect of imageability was $\mathrm{Zr}=$ $.055,95 \% \mathrm{CI}[0.035,0.075], R^{2}=.003$, or in terms of the original part correlations, $s r=.055, R^{2}=$ .003 . That is, $0.30 \%$ of the variance in word recognition performance could be explained by imageability.

Introducing the dependent variable (accuracy vs. RT) as a moderator reduced overall heterogeneity somewhat $\left(\mathrm{I}^{2}=78.26,95 \% \mathrm{CI}=[65.74,88.13]\right)$. When examining separate effects on each dependent variable, heterogeneity was still very high for both measures but greater for accuracy $\left(\mathrm{I}^{2}=84.64 \%, 95 \% \mathrm{CI}=[74.47,93.57]\right)$ than for $\mathrm{RT}\left(\mathrm{I}^{2}=63.53,95 \% \mathrm{CI}=[26.09,85.20]\right)$. As shown in Figure 7, there was a meta-effect on accuracy at $0.99 \%$ of variance $(\mathrm{Zr}=.100,95 \%$ CI [0.078, $0.122] ; R^{2}=.010$, or in terms of the original part correlations, $\left.s r=.099, R^{2}=.010\right)$, but not RT $(\mathrm{Zr}=$ $.008,95 \% \mathrm{CI}[-0.006,0.023] ; R^{2}=.000$, or in terms of the original part correlations, $s r=.008, R^{2}=$ .000). Overall, these analyses show that imageability has a small facilitatory meta-effect on accuracy but not on latency of word recognition. The effects are smaller than typically reported in the literature: lexical decision studies find that imageability explains from $1.96 \%$ of unique variance in accuracy (Tillotson et al. 2008, for high imageability words), to 3.20\% (Pexman et al., 2008), and even up to 4.50\% (Cortese \& Schock, 2013). Similarly, imageability explains between 1.44\% (Buchanan et al., 2001) to $3.10 \%$ (Adelman et al., 2006) of unique variance in lexical decision RT, and up to $4.20 \%$ of unique variance in RT on word naming (for low frequency words; Strain \& Herdman, 1999). Nonetheless, the high heterogeneity suggests that the different studies might not be tapping into the same population effect size (i.e., that of imageability on word recognition) and so the imageability meta-effect should be treated with caution as an estimate of the true effect size. 


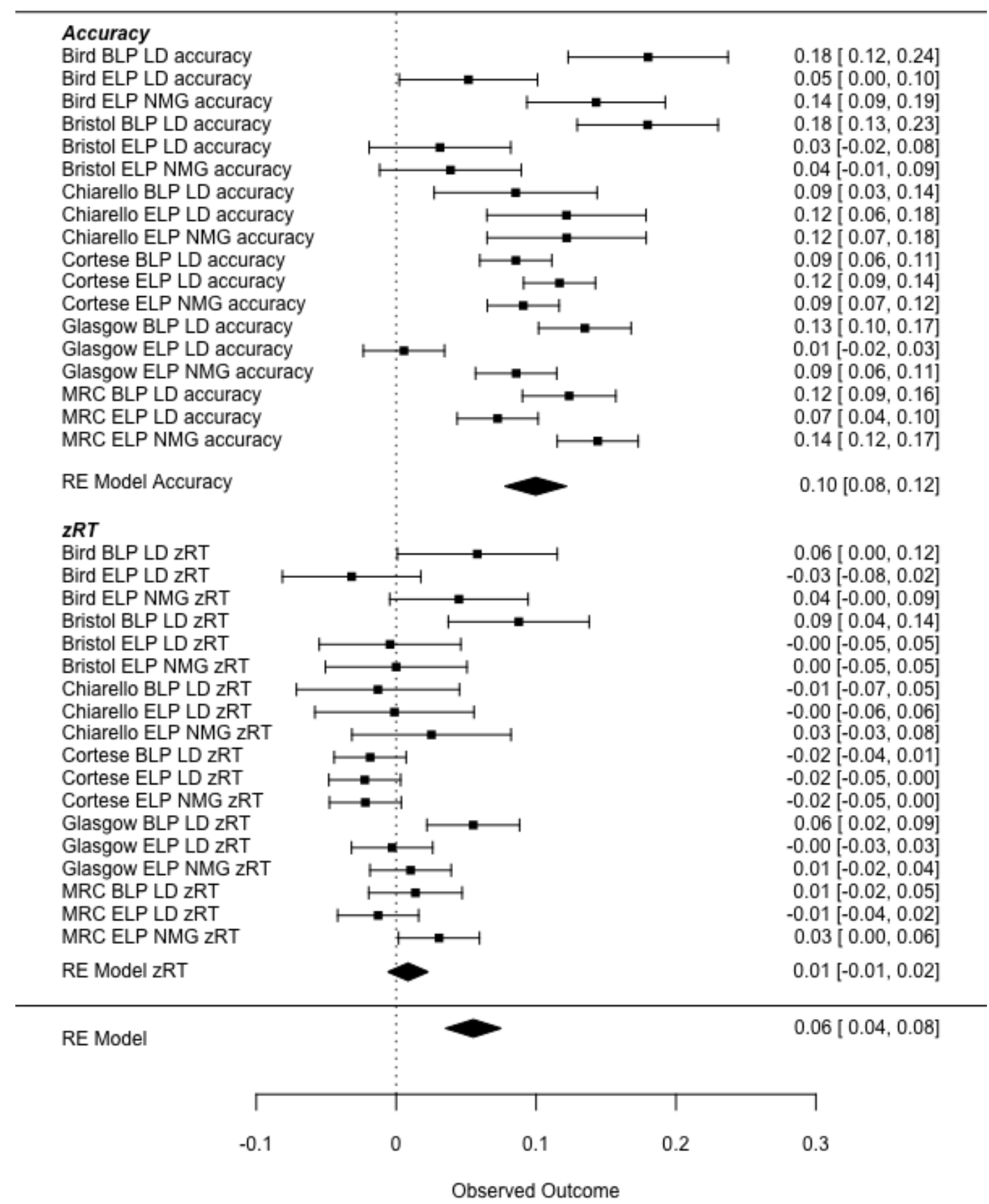

Figure 7: Forest plot of imageability effect sizes per DV in word recognition, with separate metaeffect sizes for accuracy and zRT and the overall meta-effect. 


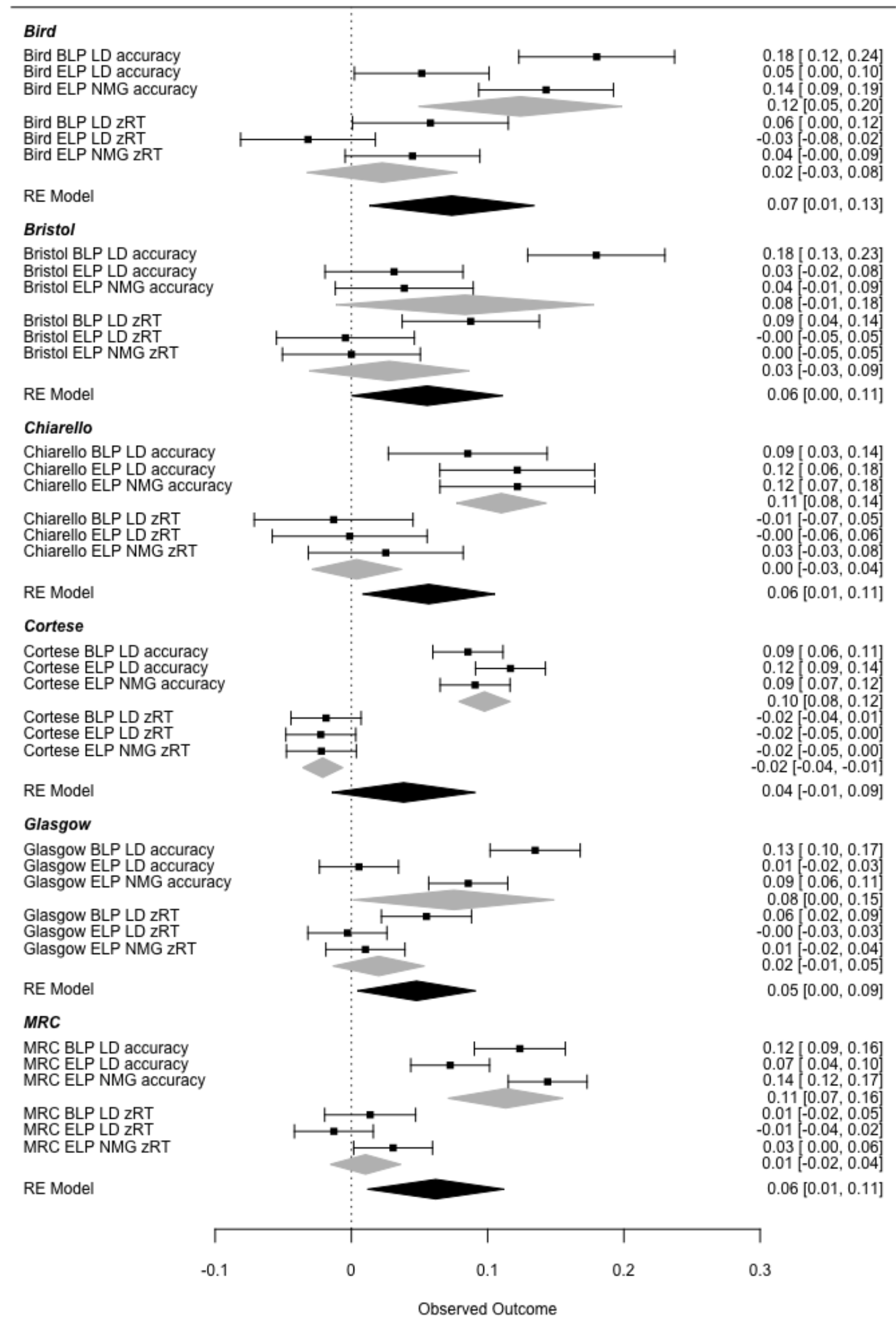

Figure 8: Forest plot of imageability effect sizes on word recognition, with meta-effect sizes per DV (grey diamonds) and overall (black diamonds) for each set of norms. "RE" refers to Restricted Maximum Likelihood. 
The forest plot in Figure 8 shows the different meta-effect sizes per set of norms, both overall and per DV. The meta-effect of the Cortese norms did not differ from zero, explaining only $0.15 \%$ variance in word recognition performance, due to the unusual combination of a clear facilitatory effect on accuracy but an inhibitory effect on RT. The Glasgow and Bristol norms produced slightly larger meta-effects (explaining $0.23 \%$ and $0.31 \%$ variance), but - as can be seen in Figure 8 - the individual estimates per DV either span or approach zero, which means their ability to facilitate word recognition should be treated with caution. The other three norms produced more robust meta-effects of facilitation, where effect sizes ranged from $0.32 \%$ and $0.38 \%$ (Chiarello and MRC norms) to $0.54 \%$ (Bird norms); these facilitation effects were true for accuracy but not for RT.

However, it is also important to examine the heterogeneity of imageability effects per set of norms (see Table 11). While heterogeneity was high for all norms in unmoderated meta-analysis, the pattern differed greatly when DV was introduced as a moderator. The heterogeneity for some norms changed very little (Glasgow and Bristol), indicating that the distinction between DVs had little to do with the variance of imageability effects between analyses. Heterogeneity remained high for some norms (Bird and MRC), indicating that while their imageability effects are indeed larger for accuracy than RT, that difference explains at best a minority of the variability in effect sizes. Two sets of norms, however, saw their heterogeneity drop to zero with addition of the DV moderator (Chiarello and Cortese), albeit with a high degree of uncertainty (i.e., wide 95\% CI around $\mathrm{I}^{2}$ ), suggesting that almost all the variability in their effect sizes may be due to the disparity between accuracy versus RT effects. Taken together, these findings suggest that no set of imageability norms can reliably facilitate RT (indeed, the only reliably meta-effect on RT was the unexpected inhibitory effect by the Cortese norms), but most (apart from the Bristol norms) can facilitate accuracy. Critically, the high degree of heterogeneity in effects, and high degree of uncertainty even when heterogeneity is low, means that even these meta-effect estimates should be considered relatively volatile. 
Table 11: Percentage of heterogeneity in meta-analysis for each set of norms, both excluding and including DV as a moderator.

\begin{tabular}{|c|c|c|c|c|}
\hline \multirow[b]{2}{*}{ Imageability norms } & \multicolumn{2}{|c|}{ Unmoderated } & \multicolumn{2}{|c|}{ DV moderator } \\
\hline & $\mathrm{I}^{2}$ & $\begin{array}{c}\text { 95\% Confidence } \\
\text { interval }\end{array}$ & $\mathrm{I}^{2}$ & $\begin{array}{c}\text { 95\% Confidence } \\
\text { interval }\end{array}$ \\
\hline Bird & 87.83 & {$[25.88,95.57]$} & 79.19 & {$[43.33,97.48]$} \\
\hline Bristol & 86.17 & {$[20.58,94.70]$} & 86.29 & {$[61.84,98.34]$} \\
\hline Chiarello & 76.92 & {$[0.00,92.38]$} & 0.00 & {$[0.00,74.93]$} \\
\hline Cortese & 89.05 & {$[75.18,98.45]$} & 0.00 & {$[0.00,85.27]$} \\
\hline Glasgow & 91.91 & {$[0.00,93.36]$} & 90.72 & {$[73.96,98.88$} \\
\hline MRC & 84.41 & {$[78.04,98.62]$} & 75.06 & {$[26.94,96.85]$} \\
\hline
\end{tabular}

\section{Conclusion}

The meta-analyses of "pure" imageability effects overall confirmed that the ease of generating a mental image for a concept facilitates performance in word recognition, but with a very small effect size $(0.30 \%$ variance) that is subject to such high statistical heterogeneity that it cannot be considered robust. Notably, our exploration of potential moderators (see Method) did not reveal any systematic differences in effect size between tasks (lexical decision vs. word naming), source of word recognition data (BLP vs. ELP), or geographic congruence of the imageability norms and word recognition data (congruent vs. incongruent), so they cannot explain why imageability effect sizes varied so much. The variability in imageability effect sizes could be explained to a limited extent by the dependent variable being analysed (i.e., accuracy vs. RT). This moderator effect supported our conclusion from Study 4 that ease of generating mental imagery does not contribute to speed of response in a word recognition task, when lexical and sensorimotor sources of variance are accounted for, even though it may contribute to accuracy of performance.

Moreover, not all sets of imageability norms produced reliable meta-effects on word recognition: the effects either did not differ from zero, or were subject such high heterogeneity (or uncertainty about heterogeneity) that it is unclear how robustly they would elicit imageability effects 
in future word recognition studies. Overall, the meta-analysis results suggest that "pure" imageability (i.e., the ease of generating a mental image, independent of lexical characteristics and sensorimotor grounding) does not facilitate word recognition in a reliable manner, and that imageability independently of lexical and sensorimotor information - makes a weak and unreliable contribution to word recognition.

\section{General discussion}

In this paper, our goal was to examine the construct of imageability, what exactly it measures relative to other sensorimotor variables commonly used in studies of semantic involvement in word recognition, and thereby investigate the extent to which imageability ratings can reliably contribute to word recognition performance. We found that imageability effects on word recognition were highly variable across different sets of norms even when the baseline model was held constant (Study 1), and that a large proportion of the variance in imageability ratings can be explained by lexical and sensorimotor information but again with differences between the norms (Study 2). When lexical and sensorimotor effects were removed from word recognition (Study 3), imageability effects remained small and inconsistent across different sets of norms (Study 4), with very small meta-effects on word recognition that come with high levels of heterogeneity (Study 5). While Study 5 showed that some of the heterogeneity of effects can be attributed to imageability differentially supporting accuracy but not speed of word recognition, variability across multiple tasks and sources of data persisted even when lexical and sensorimotor word characteristics were accounted for. That is, the pure ease of generating a mental image appears to have a weak and unreliable effect on lexical decision and word naming performance, and the discrepant results for different sets of norms suggests that imageability ratings do not reflect a reliable and stable construct that facilitates word recognition.

The results allow us to disentangle the extent to which imageability itself - that is, a construct that measures the ease of generating conscious imagery of a word - facilitates word recognition independently of the effects of sensorimotor grounding. Words vary in how they are grounded in diverse aspects of sensorimotor experience (Body, Food, Communication, Objects), all of which influence word recognition to some extent (Study 3). Once this complex sensorimotor grounding is 
accounted for, imageability ratings then sometimes, but inconsistently, predict a small amount of variance in how accurately, though not how quickly, a word is recognised (Study 4). Such systematically inconsistent imageability effects mean the present paper supports apparently contradictory findings in the literature regarding the relative abilities of sensorimotor grounding and imageability to predict word recognition. When the baseline model is held constant with a wide range of lexical information including age of acquisition and sensorimotor information across multiple dimensions, imageability sometimes has no additional effect on word recognition (supporting Connell \& Lynott, 2012a; Speed \& Brysbaert, 2021; Vergallito et al., 2020, for English data) and sometimes it does (supporting Khanna \& Cortese, 2021; Vergallito et al., 2020, for Italian data), depending on the particular set of imageability norms used. The variability in "pure" imageability effect sizes indicates that there are inherent differences between sets of imageability ratings which affect their ability to predict word recognition performance. Across tasks (lexical decision and word naming) and dependent variables (RT and accuracy), the Bird imageability norms produced the largest effects, followed by the MRC and Cortese norms, then the Chiarello norms, while the Glasgow and Bristol norms did not produce reliable effects. Their poor performance could mean that the Glasgow and Bristol imageability norms somehow do not tap into the construct of imageability at all, and instead reflect only lexical, sensorimotor, and other information not useful to word recognition. We note that the meta-effect size per set of imageability norms in Study 5 is inversely related to the proportion of variance in imageability ratings explained by the lexical and sensorimotor components in Study 2 ( $r=$ -.384 across the 6 sets of norms). Indeed, if we concentrate on the contribution of the sensorimotor components alone ${ }^{8}$, the relationship becomes markedly stronger ( $r=-.698$ across 6 sets of norms). That is, the more imageability ratings reflect sensorimotor experience of Objects, Body, Communication, and Food, the weaker the "pure" imageability effect on word recognition once this sensorimotor information has already been taken into account. Since these sensorimotor components include information from vision (particularly the Object component, but also Body), and facilitated word recognition (Study 3), it is likely that some portion of typical imageability effects is due to

\footnotetext{
8 By summing the squares of the part correlations per sensorimotor component, as displayed in Figure 2.
} 
attentional modulation in a visual task (Connell \& Lynott, 2014b, 2016b). Nonetheless, given that the imageability effects on word recognition remained variable in Studies 4 and 5 even after effects of lexical and sensorimotor information were removed, sensorimotor grounding alone is not sufficient to explain the variable patterns of imageability effects.

Interestingly, it does not appear that variable imageability effects between different norms can be explained by the variable proportion of nouns in particular subsets of items, despite previous findings that imageability ratings are higher for nouns (Bird et al., 2001; Simonsen et al., 2013) and that highly imageable nouns, but not verbs, are easier to process (Bedny \& Thompson-Schill, 2006). All norms contained between $58 \%$ and $70 \%$ of nouns (see supplemental materials), and were strongly predicted by the Object component (which incorporates a noun vs. non-noun variable) in Study 2. More specifically, the Bird norms, which elicited the strongest imageability effects in the present study, included 58\% nouns; while the Bristol and Glasgow norms, which elicited the weakest and most variable imageability effects, included $60 \%$ and $66 \%$ nouns respectively. The highest proportion of nouns were in the MRC norms (70\%), which elicited fairly reliable, but not the strongest, effects. Hence, "pure" imageability effects on word recognition are unlikely to be an artefact of our experience with noun concepts, and the inconsistent effects of different imageability norms require another explanation.

We also considered whether the variability in performance could be attributed to incongruence between the country or region where the imageability norms were collected, and that where the word recognition data were collected. That is, it might have been the case that imageability ratings from the UK would be more efficient in predicting word recognition performance in UK participants than in US participants, and vice versa. However, it was not the case: as matching the country of origin for imageability ratings and word recognition data did not systematically moderate imageability effect sizes (Study 5). While there are indeed differences between norming studies in how participants rate imageability, it is not geographic variation within or between countries that drive these differences. Nonetheless, given the findings of Vergallito et al. (2020) that the relative ability of perceptual strength to outperform imageability may vary across languages, future research 
should examine the effects of imageability on word recognition in languages other than English using a similar systematic approach to comparison different sources of imageability norms to the one we report in the present paper.

Two possible explanations of the current pattern of results remain. When lexical and sensorimotor variance is accounted for, the best-performing imageability norms, such as the Bird norms, do well in predicting word recognition because they succeed in tapping into the construct of imageability in a way that other norms (in particular the Glasgow and Bristol norms) do not, which suggests that - given that all norming studies used the same instructions and scales - the construct of imageability itself cannot be reliably rated. Alternatively, poorer performance of the Glasgow and Bristol imageability norms could mean that they do indeed reflect the ease of generating a mental image, but that this imageability construct is not a useful predictor of word recognition once lexical and sensorimotor information has been taken into account. If this is the case, then it means that the construct of imageability itself does not facilitate word recognition, and that many imageability effects previously attributed to the ease of generating a mental image are instead more likely to be due to lexical properties of the word and/or sensorimotor grounding of the referent concept. Moreover, this also means that the best-performing imageability norms, such as the Bird norms, do well in predicting word recognition for a reason other than the ease of generating a mental image, which suggests that some - but not all - imageability ratings reflect another hitherto unidentified word property that is not imageability per se. The present data cannot distinguish whether some imageability norms succeed in producing "pure" imageability effects because they have better construct validity (i.e., other imageability ratings fail to capture ease of generating a mental image for a word) or because they actually capture some other semantic construct that is useful to word recognition (i.e., all norms capture ease of generating a mental image for a word but that is not what facilitated word recognition in Study 4). Turning our attention to imageability effects in other tasks, such as word memory, may shed more light on which explanation is more likely; we are currently investigating this possibility. 
The findings reported in this paper have several methodological implications for researchers interested in semantic effects on word recognition. Firstly, we suggest that it may be necessary to use multiple sources of imageability norms in study designs, to ensure internal replication and consistent effects. In particular, caution is advised when using norms which have been demonstrated to elicit very weak or conflicting effects in the current study, namely the Bristol and Cortese norms. Another important implication is that imageability does not work as a proxy for semantic effects in word recognition, especially on speed of response. Indeed, consciously generating mental imagery and simulating sensorimotor information are two different processes, and imagery abilities do not predict the strength of effects of sensory simulation on semantic processing (Muraki \& Pexman, 2021; Pecher et al., 2009). Given the different ways that each set of imageability norms related to lexical information and sensorimotor experience in Study 2, imageability should not be treated as a placeholder for sensorimotor word content, or a catch-all variable responsible for word meaning. High heterogeneity between norms in predicting word recognition also indicates a lack of precision in what imageability represents. When only some of the norms are able to capture something over and above lexical and sensorimotor word content, despite using the same method to obtain the ratings, then imageability as a construct cannot be trusted to be reliably tested.

Another methodological issue is the inability of humans to rate information from all senses together (Connell \& Lynott, 2012a; Juhasz et al., 2011), and that is a limitation that cannot be overcome by simply asking participants to try harder. We found that the contribution of sensorimotor experience to word recognition is complex, and that it is important to consider different dimensions of sensorimotor grounding individually when testing their contribution to conceptual processing. These findings indicate that the focus should change to more comprehensive, multi-variable measures of semantic information, such as individual aspects of sensorimotor strength (see Lynott et al., 2020) alongside age of acquisition (e.g., Kuperman et al., 2012). Alternatively, imageability could continue to be used - with awareness of its limitations and its focus on visual experience with concrete, manipulable objects - in conjunction with other variables that capture complementary aspects of semantic experience, such as body-object interaction (Bennett et al., 2011; Muraki \& Pexman, 2021) 
or the non-visual aspects of sensorimotor strength, again alongside age of acquisition. In both cases, future research should also consider the utility of including emotional affect (Citron et al., 2014; Kousta et al., 2009; Kuperman et al., 2014).

In summary, the results do not fully support the claim that ease of generating a mental image reliably predicts word recognition. It is more likely that inconsistent imageability effects result from something inherent to the ratings in each norming study. Across a series of studies where lexical and sensorimotor variables were accounted for, we found that the effects of imageability varied greatly between different sets of norms. This variation could not be explained by differences in word sampling or lexico-semantic word characteristics between the norming studies; since they all used the same instructions and procedure, the variance can most likely be attributed to differences in participant strategies when rating ease of imageability. A meta-analysis of imageability effects suggested that overall imageability contributed very little to word recognition compared to previously reported results in the literature, and showed such high heterogeneity between studies that it makes questionable the existence of general imageability effect on word recognition. Overall, the findings suggest that the imageability ratings should be used with caution in word recognition research. 


\section{References}

Adelman, J. S., Brown, G. D. A., \& Quesada, F. (2006). Contextual diversity, not word frequency, determines word-naming and lexical decision times. Psychological Science, 17(9), 814-823. https://doi.org/10.1111/j.1467-9280.2006.01787.x

Bakhtiar, M., \& Weekes, B. (2014). Lexico-semantic effects on word naming in Persian: Does age of acquisition have an effect? Memory and Cognition, 43(2), 298-313. https://doi.org/10.3758/s13421-014-0472-4

Balota, D. A., Cortese, M. J., Sergent-Marshall, S. D., Spieler, D. H., \& Yap, M. J. (2004). Visual word recognition of single-syllable words. Journal of Experimental Psychology: General, 133(2), 283316. https://doi.org/10.1037/0096-3445.133.2.283

Balota, D. A., Yap, M. J., Hutchison, K. A., Cortese, M. J., Kessler, B., Loftis, B., Nelson, D. L., Simpson, G. B. \& Treiman, R. (2007). The English lexicon project. Behavior Research Methods, 39(3), 445-459. https://doi.org/10.3758/bf03193014

Barsalou, L. W., Santos, A., Simmons, W. K., \& Wilson, C. D. (2008). Language and simulation in conceptual processing. In M. De Vega, A. M. Glenberg, and A. C. Graesser (Eds), Symbols, embodiment, and meaning (pp. 246-283). Oxford: Oxford University Press.

Bedny, M., \& Thompson-Schill, S. L. (2006). Neuroanatomically separable effects of imageability and grammatical class during single-word comprehension. Brain and Language, 98(2), 127-139. https://doi.org/10.1016/j.bandl.2006.04.008

Bennett, S. D. R., Burnett, A. N., Siakaluk, P. D., \& Pexman, P. M. (2011). Imageability and body-object interaction ratings for 599 multisyllabic nouns. Behavior Research Methods, 43(4), 1100-1109. https://doi.org/10.3758/s13428-011-0117-5

Bird, H., Franklin, S., \& Howard, D. (2001). Age of acquisition and imageability ratings for a large set of words, including verbs and function words. Behavior Research Methods, Instruments, \& Computers, 33(1), 73-79. https://doi.org/10.3758/BF03195349

Brysbaert, M., Mandera, P., \& Keuleers, E. (2018). The word frequency effect in word processing: An updated review. Current Directions in Psychological Science, 27(1), 45-50. https://doi.org/10.1177/0963721417727521 
Brysbaert, M., Van Wijnendaele, I., \& De Deyne, S. (2000). Age-of-acquisition effects in semantic processing tasks. Acta Psychologica, 104(2), 215-226. https://doi.org/10.1016/S00016918(00)00021-4

Buchanan, L., Westbury, C., \& Burgess, C. (2001). Characterizing semantic space: Neighborhood effects in word recognition. Psychonomic Bulletin and Review, 8(3), 531-544. https://doi.org/10.3758/BF03196189

Campos, A., \& Sueiro, E. (1991). Stability of imagery scores. Perceptual and Motor Skills, 72(2), 411414. https://doi.org/10.2466/pms.1991.72.2.411

Chedid, G., Brambati, S. M., Bedetti, C., Rey, A. E., Wilson, M. A., \& Vallet, G. T. (2019). Visual and auditory perceptual strength norms for 3,596 French nouns and their relationship with other psycholinguistic variables. Behavior Research Methods, 51(5), 2094-2105. https://doi.org/10.3758/s13428-019-01254-w

Chiarello, C., Shears, C., \& Lund, K. (1999). Imageability and distributional typicality measures of nouns and verbs in contemporary English. Behavior Research Methods, Instruments, \& Computers, 31(4), 603-637. https://doi.org/10.3758/BF03200739

Citron, F. M. M., Weekes, B. S., \& Ferstl, E. C. (2014). Arousal and emotional valence interact in written word recognition. Language, Cognition and Neuroscience, 29(10), 1257-1267. https://doi.org/10.1080/23273798.2014.897734

Clark, J. M., \& Paivio, A. (2004). Extensions of the Paivio, Yuille, and Madigan (1968) norms. Behavior Research Methods, Instruments, \& Computers, 36(3), 371-383. https://doi.org/10.3758/BF03195584

Coltheart, M. (1981). The MRC psycholinguistic database. Quarterly Journal of Experimental Psychology, 33(4), 497-505. https://doi.org/10.1080\%2F14640748108400805

Coltheart, M., Rastle, K., Perry, C., Langdon, R., \& Ziegler, J. (2001). DRC: a dual route cascaded model of visual word recognition and reading aloud. Psychological review, 108(1), 204. https://doi/10.1037/0033-295X.108.1.204

Connell, L. (2018). What have labels ever done for us? The linguistic shortcut in conceptual processing. Language, Cognition and Neuroscience, 34(10), 1308-1318. 
https://doi.org/10.1080/23273798.2018.1471512

Connell, L., \& Lynott, D. (2012a). Strength of perceptual experience predicts word processing performance better than concreteness or imageability. Cognition, 125(3), 452-465. https://doi.org/10.1016/j.cognition.2012.07.010

Connell, L., \& Lynott, D. (2012b). When does perception facilitate or interfere with conceptual processing? The effect of attentional modulation. Frontiers in Psychology, 3, 1-4. .org/10.3389/fpsyg.2012.00474

Connell, L., \& Lynott, D. (2014a). Principles of representation: Why you can't represent the same concept twice. Topics in Cognitive Science, 6, 390-406. https://doi.org/10.1111/tops.12097

Connell, L., \& Lynott, D. (2014b). I see/hear what you mean: Semantic activation in visual word recognition depends on perceptual attention. Journal of Experimental Psychology: General, 143(2), 527-533. https://doi.org/10.1037/a0034626

Connell, L., \& Lynott, D. (2016a). Do we know what we're simulating? Information loss on transferring unconscious perceptual simulation to conscious imagery. Journal of Experimental Psychology: Learning Memory and Cognition, 42(8), 1218-1232. https://doi.org/10.1037/xlm0000245

Connell, L., \& Lynott, D. (2016b). Embodied semantic effects in visual word recognition. In M. H. Fischer \& Y. Coello (Eds.), Foundations of embodied cognition (Vol. 2, pp. 71-92). Hove, UK: Psychology Press.

Connell, L., Lynott, D., \& Dreyer, F. (2012). A functional role for modality-specific perceptual systems in conceptual representations. PLoS One, 7, e33321. https://doi.org/10.1371/journal.pone.0033321

Cortese, M. J., \& Fugett, A. (2004). Imageability ratings for 3,000 monosyllabic words. Behavior Research Methods, Instruments, \& Computers, 36(3), 384-387. https://doi.org/10.3758/BF03195585

Cortese, M. J., \& Khanna, M. M. (2007). Age of acquisition predicts naming and lexical-decision performance above and beyond 22 other predictor variables: An analysis of 2,342 words. Quarterly Journal of Experimental Psychology, 60(8), 1072-1082.

Cortese, M. J., Khanna, M. M., \& Hacker, S. (2010). Recognition memory for 2,578 monosyllabic words. 
Memory, 18(6), 595-609. https://doi.org/10.1080/09658211.2010.493892

Cortese, M. J., McCarty, D. P., \& Schock, J. (2015). A mega recognition memory study of 2897 disyllabic words. Quarterly Journal of Experimental Psychology, 68(8), 1489-1501. https://doi.org/10.1080/17470218.2014.945096

Cortese, M. J., \& Schock, J. (2013). Imageability and age of acquisition effects in disyllabic word recognition. Quarterly Journal of Experimental Psychology, 66(5), 946-972. https://doi.org/10.1080/17470218.2012.722660

Cumming, G. (2012). Understanding the new statistics effect sizes, confidence intervals, and metaanalysis ( $1^{\text {st }}$ ed., Multivariate applications book series). New York: Routledge.

de Groot, A. M. (1989). Representational aspects of word imageability and word frequency as assessed through word association. Journal of Experimental Psychology: Learning, Memory, and Cognition, 15, 824-845. https://doi.org/10.1037/0278-7393.15.5.824

Ellis, A. W., \& Monaghan, J. (2002). Reply to Strain, Patterson and Seidenberg (2002). Journal of Experimental Psychology: Learning, Memory, and Cognition, 28(1), 215-220. https://doi.org/10.1037/0278-7393.28.1.215

Estes, Z., Verges, M., \& Adelman, J. S. (2015). Words, objects, and locations: Perceptual matching explains spatial interference and facilitation. Journal of Memory and Language, 84, 167-189. https://doi.org/10.1016/j.jml.2015.06.002

Evans, G. A. L., Ralph, M. A. L., \& Woollams, A. M. (2012). What's in a word? A parametric study of semantic influences on visual word recognition. Psychonomic Bulletin and Review, 19(2), 325331. https://doi.org/10.3758/s13423-011-0213-7

Ferrand, L., Brysbaert, M., Keuleers, E., New, B., Bonin, P., Méot, A., ... Pallier, C. (2011). Comparing word processing times in naming, lexical decision, and progressive demasking: evidence from chronolex. Frontiers in Psychology, 2, 306. https://doi.org/10.3389/fpsyg.2011.00306

Foxe, J. J., Simpson, G. V., Ahlfors, S. P., \& Saron, C. D. (2005). Biasing the brain's attentional set: I. Cue driven deployments of intersensory selective attention. Experimental Brain Research, 166, 370-392. https://doi.org/10.1007/s00221-005-2378-7

Gilhooly, K. J., \& Logie, R. H. (1980). Age-of-acquisition, imagery, concreteness, familiarity, and 
ambiguity measures for 1,944 words. Behavior Research Methods \& Instrumentation, 12(4), 395-427. https://doi.org/10.3758/BF03201693

González-Nosti, M., Barbón, A., Rodríguez-Ferreiro, J., \& Cuetos, F. (2014). Effects of the psycholinguistic variables on the lexical decision task in Spanish: A study with 2,765 words. Behavior Research Methods, 46(2), 517-525. https://doi.org/10.3758/s13428-013-0383-5

Grandy, T. H., Lindenberger, U., \& Schmiedek, F. (2020). Vampires and nurses are rated differently by younger and older adults-Age-comparative norms of imageability and emotionality for about 2500 German nouns. Behavior Research Methods, 52(3), 1-10. https://doi.org/10.3758/s13428019-01294-2

Harm, M. W., \& Seidenberg, M. S. (2004). Computing the meanings of words in reading: cooperative division of labor between visual and phonological processes. Psychological review, 111(3), 662. https://psycnet.apa.org/doi/10.1037/0033-295X.111.3.662

Hoffman, P., Ralph, M. A. L., \& Rogers, T. T. (2013). Semantic diversity: A measure of semantic ambiguity based on variability in the contextual usage of words. Behavior Research Methods, 45(3), 718-730. https://doi.org/10.3758/s13428-012-0278-X

James, C. T. (1975). The role of semantic information in lexical decisions. Journal of Experimental Psychology: Human Perception and Performance, 1(2), 130-136.

JASP Team (2020). JASP (Version 0.14.1)[Computer software].

Juhasz, B. J., Yap, M. J., Dicke, J., Taylor, S. C., \& Gullick, M. M. (2011). Tangible words are recognized faster: The grounding of meaning in sensory and perceptual systems. Quarterly Journal of Experimental Psychology, 64(9), 1683-1691. https://doi.org/10.1080/17470218.2011.605150

Keuleers, E., Lacey, P., Rastle, K., \& Brysbaert, M. (2012). The British lexicon project: Lexical decision data for 28,730 monosyllabic and disyllabic English words. Behavior Research Methods, 44(1), 287-304. https://doi.org/10.3758/s13428-011-0118-4

Khanna, M. M., \& Cortese, M. J. (2021). How well imageability, concreteness, perceptual strength, and action strength predict recognition memory, lexical decision, and reading aloud performance. Memory, 1-15. https://doi.org/10.1080/09658211.2021.1924789 
Kousta, S. T., Vinson, D. P., \& Vigliocco, G. (2009). Emotion words, regardless of polarity, have a processing advantage over neutral words. Cognition, 112(3), 473-481. https://doi.org/10.1016/j.cognition.2009.06.007

Kuperman, V., Estes, Z., Brysbaert, M., \& Warriner, A. B. (2014). Emotion and language: valence and arousal affect word recognition. Journal of Experimental Psychology: General, 143, 1065-1081.

Kuperman, V., Stadthagen-Gonzalez, H., \& Brysbaert, M. (2012). Age-of-acquisition ratings for 30,000 English words. Behavior Research Methods, 44(4), 978-990

Langner, R., Kellermann, T., Boers, F., Sturm, W., Willmes, K., \& Eickhoff, S. B. (2011). Modalityspecific perceptual expectations selectively modulate baseline activity in auditory, somatosensory, and visual cortices. Cerebral Cortex, 21, 2850-2862. https://doi.org/10.1093/cercor/bhr083

Lipsey, M., \& Wilson, D. B. (2001). Practical meta-analysis. Thousand Oaks, CA: SAGE.

Lynott, D., Connell, L., Brysbaert, M., Brand, J., \& Carney, J. (2020). The Lancaster Sensorimotor Norms: multidimensional measures of perceptual and action strength for 40,000 English words. Behavior Research Methods, 52, 1-21.

Muraki, E. J., \& Pexman, P. M. (2021). Simulating Semantics: Are Individual Differences in Motor Imagery Related to Sensorimotor Effects in Language Processing?. Retrieved from PsyArXiv database. https://doi.org/10.31234/osf.io/m5ge3

Paivio, A. (1971). Imagery and language. In S. J. Segal (Ed.), Imagery: Current cognitive approaches. (pp. 7-32). New York: Academic Press.

Paivio, A. (1990). Mental representations: A dual coding approach. Oxford University Press.

Paivio, A., Yuille, J. C., \& Madigan, S. A. (1968). Concreteness, imagery, and meaningfulness values for 925 nouns. Journal of Experimental Psychology, 76(1), 1-12. https://psycnet.apa.org/doi/10.1037/h0025327

Palmiero, M., Olivetti Belardinelli, M., Nardo, D., Sestieri, C., Di Matteo, R., D’Ausilio, A., \& Romani, G. L. (2009). Mental imagery generation in different modalities activates sensory-motor areas. Cognitive Processing, 10, 268-271. http://dx.doi.org/10.1007/s10339-009-0324-5

Pecher, D., van Dantzig, S., \& Schifferstien, H. N. J. (2009). Concepts are not represented by conscious 
imagery. Psychonomic Bulletin and Review, 16(5), 914-919.

https://doi.org/10.3758/PBR.16.5.914

Pexman, P. M., Hargreaves, I. S., Siakaluk, P. D., Bodner, G. E., \& Pope, J. (2008). There are many ways to be rich: Effects of three measures of semantic richness on visual word recognition. Psychonomic Bulletin and Review, 15(1), 161-167. https://doi.org/10.3758/PBR.15.1.161

R Core Team (2013). R: A language and environment for statistical computing. R Foundation for Statistical Computing, Vienna, Austria. http://www.R-project.org/.

Revelle, W. (2021) psych: Procedures for Personality and Psychological Research, Northwestern University, Evanston, Illinois, USA, https://CRAN.R-project.org/package=psych.

Sabsevitz, D. S., Medler, D. A., Seidenberg, M., \& Binder, J. R. (2005). Modulation of the semantic system by word imageability. Neuroimage, 27(1), 188-200. https://doi.org/10.1016/j.neuroimage.2005.04.012

Sadoski, M. \& Paivio, A. (2004). A dual coding theoretical model of reading. In R. B. Ruddell \& N. J. Unrau (Eds.), Theoretical models and processes of reading (5th ed., pp. 1329-1362). Newark, DE: International Reading Association.

Schock, J., Cortese, M. J., \& Khanna, M. M. (2012). Imageability estimates for 3,000 disyllabic words. Behavior Research Methods, 44(2), 374-379. https://doi.org/10.3758/s13428-011-0162-0

Scott, G. G., Keitel, A., Becirspahic, M., Yao, B., \& Sereno, S. C. (2018). The Glasgow norms: Ratings of 5,500 words on nine scales. Behavior Research Methods, 51(3), 1258-1270. https://doi.org/10.3758/s13428-018-1099-3

Shaoul, C., \& Westbury, C. (2010). Exploring lexical co-occurrence space using HiDEx. Behavior Research Methods, 42(2), 393-413. https://doi.org/10.3758/BRM.42.2.393

Siakaluk, P. D., Newcombe, P. I., Duffels, B., Li, E., Sidhu, D. M., Yap, M. J., \& Pexman, P. M. (2016). Effects of emotional experience in lexical decision. Frontiers in Psychology, 7, article 1157. https://doi.org/10.3389/fpsyg.2016.01157

Sidhu, D. M., Kwan, R., Pexman, P. M., \& Siakaluk, P. D. (2014). Effects of relative embodiment in lexical and semantic processing of verbs. Acta psychologica, 149, 32-39. https://doi.org/10.1016/j.actpsy.2014.02.009 
Simonsen, H. G., Lind, M., Hansen, P., Holm, E., \& Mevik, B. H. (2013). Imageability of Norwegian nouns, verbs and adjectives in a cross-linguistic perspective. Clinical Linguistics and Phonetics, 27(6-7), 435-446. https://doi.org/10.3109/02699206.2012.752527

Speed, L.J., Brybaert, M. (2021) Dutch sensory modality norms. Behavior Research Methods. Advance online publication. https://doi.org/10.3758/s13428-021-01656-9

Spence, C., Nicholls, M. E., \& Driver, J. (2001). The cost of expecting events in the wrong sensory modality. Perception \& Psychophysics, 63, 330-336. https://doi.org/10.3758/bf03194473

Stadthagen-Gonzalez, H., \& Davis, C. J. (2006). The Bristol norms for age of acquisition, imageability, and familiarity. Behavior Research Methods, 38(4), 598-605.

Strain, E., \& Herdman, C. M. (1999). Imageability effects in word naming: an individual differences analysis. Canadian Journal of Experimental Psychology/Revue canadienne de psychologie expérimentale, 53(4), 347. https://doi.org/10.1037/h0087322

Tillotson, S. M., Siakaluk, P. D., \& Pexman, P. M. (2008). Body—object interaction ratings for 1,618 monosyllabic nouns. Behavior Research Methods, 40(4), 1075-1078.

Toglia, M. P., \& Batti,g W. F. (1978). Handbook of semantic word norms. Hillsdale, NJ: Erlbaum. van Heuven, W. J. B., Mandera, P., Keuleers, E., \& Brysbaert, M. (2014). SUBTLEX-UK: A new and improved word frequency database for British English. Quarterly Journal of Experimental Psychology, 67(6), 1176-1190. https://doi.org/10.1080/17470218.2013.850521

Venables, W. N., \& Ripley, B. D. (2002). Random and mixed effects. In Modern applied statistics with $S$ (pp. 271-300). Springer, New York, NY.

Vergallito, A., Petilli, M. A., \& Marelli, M. (2020). Perceptual modality norms for 1,121 Italian words: A comparison with concreteness and imageability scores and an analysis of their impact in word processing tasks. Behavior Research Methods, 52(4), 1599-1616. https://doi.org/10.3758/s13428019-01337-8

Veroniki, A. A., Jackson, D., Viechtbauer, W., Bender, R., Bowden, J., Knapp, G., Kuss, O., Higgins, J. P., Langan, D., \& Salanti, G. (2016). Methods to estimate the between-study variance and its uncertainty in meta-analysis. Research Synthesis Methods, 7(1), 55-79. https://doi.org/10.1002/jrsm.1164 
Vigliocco, G., Meteyard, L., Andrews, M., \& Kousta, S. (2009). Toward a theory of semantic representation. Language and Cognition, 1(02), 219-247. https://doi.org/10.1515/LANGCOG.2009.011

Wagenmakers, EJ. A practical solution to the pervasive problems ofp values. Psychonomic Bulletin \& Review 14, 779-804 (2007). https://doi.org/10.3758/BF03194105

Westbury, C., \& Moroschan, G. (2009). Imageability x phonology interactions during lexical access: Effects of modality, phonological neighbourhood, and phonological processing efficiency. The Mental Lexicon, 4(1), 115-145. https://doi.org/10.1075/ml.4.1.05wes

Wickham, H., Averick, M., Bryan, J., Chang, W., D’Agostino McGowan, L., François, R., Grolemund, G., Hayes, A., Henry, L., Hester, J., Kuhn, M., Lin Pedersen, T., Miller, E., Milton Bache, S., Muller, K., Ooms, J., Robinson, D., Paige Seidel, D., Spinu, V. ... \& Yutani, H. (2019). Welcome to the Tidyverse. Journal of Open Source Software, 4(43), 1686. https://doi.org/10.21105/joss.01686

Wilson, M. A., Cuetos, F., Davies, R., \& Burani, C. (2013). Revisiting age-of-acquisition effects in Spanish visual word recognition: The role of item imageability. Journal of Experimental Psychology: Learning Memory and Cognition, 39(6), 1842-1859. https://doi.org/10.1037/a0033090

Wingfield, C., \& Connell, L. (2021). Understanding the role of linguistic distributional knowledge in cognition. PsyArXiv. https://doi.org/10.31234/osf.io/hpm4z

Woollams, A. M. (2005). Imageability and ambiguity effects in speeded naming: Convergence and divergence. Journal of Experimental Psychology: Learning Memory and Cognition, 31(5), 878890. https://doi.org/10.1037/0278-7393.31.5.878

Yap, M. J., Lim, G. Y., \& Pexman, P. M. (2015). Semantic richness effects in lexical decision: The role of feedback. Memory and Cognition, 43(8), 1148-1167. https://doi.org/10.3758/s13421-0150536-0

Yap, M. J., Pexman, P. M., Wellsby, M., Hargreaves, I. S., \& Huff, M. J. (2012). An abundance of riches: Cross-task comparisons of semantic richness effects in visual word recognition. Frontiers in Human Neuroscience, 6, 1-10. https://doi.org/10.3389/fnhum.2012.00072 
Yap, M. J., Tan, S. E., Pexman, P. M., \& Hargreaves, I. S. (2011). Is more always better? Effects of semantic richness on lexical decision, speeded pronunciation, and semantic classification. Psychonomic Bulletin \& Review, 18(4), 742-750.

Yates, M. (2005). Phonological neighbors speed visual word processing: Evidence from multiple tasks. Journal of Experimental Psychology: Learning Memory and Cognition, 31(6), 1385-1397. https://doi.org/10.1037/0278-7393.31.6.1385

Zevin, J. D., \& Seidenberg, M. S. (2002). Age of acquisition effects in word reading and other tasks. Journal of Memory and Language, 47(1), 1-29. 